\title{
FULLY DISCRETE SECOND-ORDER LINEAR SCHEMES FOR HYDRODYNAMIC PHASE FIELD MODELS OF BINARY VISCOUS FLUID FLOWS WITH VARIABLE DENSITIES*
}

\author{
YUEZHENG GONG ${ }^{\dagger}$, JIA ZHAO $\ddagger$ XIAOGANG YANG§ ${ }^{\S}$ AND QI WANG
}

\begin{abstract}
We develop spatial-temporally second-order, energy stable numerical schemes for two classes of hydrodynamic phase field models of binary viscous fluid mixtures of different densities. One is quasi-incompressible while the other is incompressible. We introduce a novel energy quadratization technique to arrive at fully discrete linear schemes, where in each time step only a linear system needs to be solved. These schemes are then shown to be unconditionally energy stable rigorously subject to periodic boundary conditions so that a large time step is plausible. Both spatial and temporal mesh refinements are conducted to illustrate the second-order accuracy of the schemes. The linearization technique developed in this paper is so general that it can be applied to any thermodynamically consistent hydrodynamic theories so long as their energies are bounded below. Numerical examples on coarsening dynamics of two immiscible fluids and a heavy fluid drop settling in a lighter fluid matrix are presented to show the effectiveness of the proposed linear schemes. Predictions by the two fluid mixture models are compared and discussed, leading to our conclusion that the quasiincompressible model is more reliable than the incompressible one.
\end{abstract}

Key words. phase field, hydrodynamic, energy stable schemes, linear

AMS subject classifications. 65MXX, 76TXX

DOI. $10.1137 / 17 \mathrm{M} 1111759$

1. Introduction. The phase field method, as a very popular interface capturing method, has been widely used to study a variety of interfacial dynamic problems $[7,9,10,12,17,19,20,32,36,37,38,41,46,48,49,58,59,60,61,62,63]$. Its basic idea is to employ an order parameter, or the phase variable, to distinguish distinct material phases in the multiphasic material system, which varies continuously over thin interfacial layers and is mostly uniform in bulk phases. The set of governing equations in the phase field model can be derived variationally from its energy functional guided by the generalized Onsager principle [52], where phase variables are transported by advection-diffusion-reaction equations (usually the Cahn-Hilliard

*Submitted to the journal's Computational Methods in Science and Engineering section January 17, 2017; accepted for publication (in revised form) July 6, 2017; published electronically January 30, 2018.

http://www.siam.org/journals/sisc/40-1/M111175.html

Funding: The first author's work was partially supported by the opening project of State Key Laboratory of Explosion Science and Technology (Beijing Institute of Technology) through grant KFJJ11-4M, the China Postdoctoral Science Foundation through grant 2016M591054, and the foundation of Jiangsu Key Laboratory for Numerical Simulation of Large Scale Complex Systems (201703). The second author's work was partially supported by an ASPIRE research grant from the Office of the Vice President for Research, University of South Carolina. The third author's work was partially supported by the Scientific Research Fund of Wuhan Institute of Technology through grant K201741. The fourth author's work was partially supported by grants NSF-DMS-1200487 and DMS-1517347 and NSFC awards 11571032, 91630207, and NSAF-U1530401.

${ }^{\dagger}$ College of Science, Nanjing University of Aeronautics and Astronautics, Nanjing 210016, China (gongyuezheng@nuaa.edu.cn).

${ }_{\ddagger}$ Department of Mathematics and Statistics, Utah State University, Logan, UT 84321 (jia.zhao@ usu.edu),

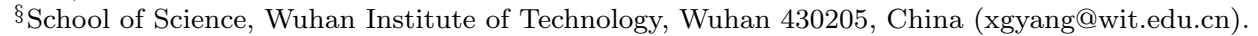

I Corresponding author. Beijing Computational Science Research Center, Beijing 100193, China, and School of Materials Science and Engineering, Nankai University, Tianjin 300350, China (qwang@csrc.ac.cn).

B138 
equation or the Allen-Cahn equation) and are coupled with the fluid momentum balance equation (Navier-Stokes equation) as well as the mass conservation equation. Compared to other mathematical and computational technologies available for studying multiphasic materials such as the front-tracking and level-set methods [35, 43, 47], the phase field method exhibits a clear advantage in its simplicity in model formulation, ease of numerical implementation, and the ability to include essential interfacial physics. The most attractive numerical feature of this method is that the interface is not tracked explicitly, but instead is implicitly defined through the level surface of the continuous phase field function.

For binary fluid mixtures with components $A$ and $B$, one commonly uses a phase variable $\phi \in[0,1]$ to represent the volume fraction of fluid $A$. The volume fraction of fluid $B$ is then given by $1-\phi$. The transition layer given by $\{\mathbf{x} \mid 0<\phi<1\}$ denotes the interfacial region between the two bulk phases. We acknowledge that there are other phase field models, developed for viscous fluids, that are formulated using mass fractions [42]. For historical reasons, most mixing energies are calculated in terms of the volume fraction instead of the mass fraction in the literature for multiphasic polymeric fluids $[18,21]$. Therefore, the phase field model with a volume fraction formulation is easier to formulate with a free energy calculated from polymer physics.

Consider a smooth domain $\Omega \subset \mathbb{R}^{2}$ occupied by the fluid mixture; the free energy of the mixture system is given by

$$
F[\phi]=\int_{\Omega}\left(\frac{\gamma_{1}}{2}|\nabla \phi|^{2}+f(\phi)\right) d \mathbf{x},
$$

where $\gamma_{1}$ is a parameter measuring the strength of the conformational entropy and $f(\phi)$ is the bulk energy density functional, which will be discussed in detail in the next section.

A classical phase field model for two incompressible, viscous fluids with the same density was introduced by Hohenberg and Halperin [34]. It consists of the following system of PDEs:

$$
\begin{aligned}
& \rho\left(\partial_{t} \mathbf{v}+\mathbf{v} \cdot \nabla \mathbf{v}\right)=-\nabla p+\nabla \cdot \tau-\phi \nabla \mu \\
& \nabla \cdot \mathbf{v}=0 \\
& \partial_{t} \phi+\nabla \cdot(\phi \mathbf{v})=\nabla \cdot(\lambda \nabla \mu),
\end{aligned}
$$

where $\mathbf{v}$ is the mass-average velocity, $p$ is the hydrostatic pressure, $\tau=2 \eta \mathbf{D}$ is the viscous stress tensor with the shear viscosity $\eta$ and the strain rate tensor $\mathbf{D}=\frac{1}{2}(\nabla \mathbf{v}+$ $\left.\nabla \mathbf{v}^{T}\right), \mu=\frac{\delta F}{\delta \phi}$ is the chemical potential given by $\mu=f^{\prime}(\phi)-\gamma_{1} \Delta \phi$, and $\lambda$ is the mobility coefficient that can be a function of phase variable $\phi$. The model (1.2) has been successfully used to simulate complicated mixing flows involving incompressible fluid components with matched densities [11]. Gurtin et al. showed thermodynamic consistency of the model [33]. Gong et al. developed a fully discrete energy stable scheme for the model [24], where the discrete energy law is conserved exactly. There are lots of energy stable schemes and convergence analyses related to model (1.2) or its simplified version in the literature. Interested readers can refer to $[4,13,14,15,16]$ for details.

However, the model (1.2) may not be used if the two incompressible fluid components have different densities. For binary fluid problems with a small density difference, a common practice is to use the Boussinesq approximation, where the small density difference is neglected except in the gravitational force [40]. This approach, 
however, is no longer valid for large density differences. Several quasi-incompressible phase field models for the case of different densities have been developed and discussed in $[3,10,17,42,44,45]$, in which the models in $[3,44]$ consider phenomenological modifications of the momentum equation. In addition, the model in [44] is not frameindifferent to rotations in the coordinate system. The thermodynamically consistent versions have been investigated analytically in $[1,2]$, where existence of strong localin-time solutions and weak solutions are shown. Benchmark computations for the models of $[3,10,17]$ were carried out by Aland and Voigt [6]. In [30], a numerical method that preserves a discrete energy law is developed for the quasi-incompressible model of [42], in which the mass fraction is used as the phase variable.

More recently, a new quasi-incompressible model for binary fluid flows with different densities was developed by Shen et al. and Aki et al. [5, 45] and for multiphasic fluid flows by Li and Wang [39] using volume fractions as phase field variables. For binary fluid mixtures, the model consists of the following coupled PDEs:

$$
\begin{aligned}
& \rho\left(\partial_{t} \mathbf{v}+\mathbf{v} \cdot \nabla \mathbf{v}\right)=-\nabla p+\nabla \cdot \tau-\phi \nabla \mu, \\
& \nabla \cdot \mathbf{v}=a \nabla \cdot(\lambda \nabla(\mu+a p)), \\
& \partial_{t} \phi+\nabla \cdot(\phi \mathbf{v})=\nabla \cdot(\lambda \nabla(\mu+a p)),
\end{aligned}
$$

where $a=1-\frac{\rho_{1}}{\rho_{2}}, \rho_{1,2}$ are constant mass densities of fluids $A$ and $B, \rho=\rho_{1} \phi+\rho_{2}(1-\phi)$ is the total mass density, and $\tau=2 \eta \mathbf{D}+\nu \operatorname{tr}(\mathbf{D}) \mathbf{I}$ with the shear viscosity $\eta=$ $\eta_{1} \phi+\eta_{2}(1-\phi)$ and the volumetric viscosity $\nu=\nu_{1} \phi+\nu_{2}(1-\phi)$. The definitions of other variables are the same as those in model (1.2). The model given in (1.3) differs from the quasi-incompressible Navier-Stokes-Cahn-Hilliard (NSCH) system developed in [42] in that the volume fraction of one fluid component, rather than the mass concentration of the component, is used as the phase variable. In addition, the two models are derived with different energy functionals. A discontinuous Galerkin finite element method [23] and a new structure-preserving algorithm [26] were developed for model (1.3), where the corresponding discrete energy dissipation law is preserved. An alternative approach was taken to modify the compressible models into an incompressible version [3], where the momentum equation is approximated using the volume-average velocity instead of the mass-average velocity. Specifically, the incompressible hydrodynamic phase field model for fluid mixtures of variable densities consists of the following equations:

$$
\begin{aligned}
& \rho \partial_{t} \mathbf{v}+\mathbf{a} \cdot \nabla \mathbf{v}=-\nabla p+\nabla \cdot \tau-\phi \nabla \mu, \\
& \nabla \cdot \mathbf{v}=0, \\
& \partial_{t} \phi+\nabla \cdot(\phi \mathbf{v})=\nabla \cdot(\lambda \nabla \mu),
\end{aligned}
$$

where $\mathbf{v}$ is the volume-average velocity, $\tau=2 \eta \mathbf{D}$ and $\mathbf{a}=\rho \mathbf{v}+\left(\rho_{2}-\rho_{1}\right) \lambda \nabla \mu$. The other variables are the same as in the above quasi-incompressible model. The model given in (1.4) has been studied numerically in [22, 28, 29], where the numerical methods developed are energy stable.

However, the numerical schemes developed so far for model (1.3) or model (1.4) in the literature are either linear, first order in time, or, even if they can reach secondorder accuracy in time, they are nonlinear so that a highly nonlinear system has to be solved iteratively $[22,28,29,30,31]$. The nonlinear systems usually require sophisticated implementation and a smaller time step to ensure convergence of the numerical solver. In this paper, we propose a novel linearization strategy (inspired by 
a recently developed energy quadratization strategy $[50,51,53,54,56,57,64,65])$ to develop fully discrete schemes that are linear and second order in time and space. The advantage of our proposed schemes includes (1) it is easy to implement (only a Krylov subspace solver is necessary); (2) the linearization strategy employed is so general that it works for both model (1.3) and model (1.4) without any restrictions on the specific form of the bulk energy; (3) the proposed schemes preserve the corresponding discrete energy dissipation law at any time-step size and thus are unconditionally energy stable. Several numerical examples, including drop dynamics of viscous fluid drops immersed in another viscous fluid matrix and mixing dynamics of binary polymeric (viscous) solutions, will be presented to show the accuracy and efficiency of the new schemes. In our numerical studies, we show that the two models in fact describe distinct transient dynamics for binary fluid mixtures of two incompressible fluid components at large density differences. In particular, the numerical examples show that model (1.4) is insensitive to the density difference while model (1.3) is sensitive to it. In addition, one can show that model (1.4) is in fact an approximation of model (1.3) by violating the balance of linear momentum [55]. We therefore believe that model (1.3) should be the choice when density differences are large. Of course, a more detailed comparison between the model and carefully designed experiments should be carried out to justify it. But this is beyond the scope of the current paper.

The model in (1.3) based on the mass-average velocity is called the PFM model, while the model in (1.4) based on the volume-average velocity is called the PFV model in this paper [55]. We note that both models satisfy an energy dissipation law. But, the kinetic energy in the PFV model is not the real kinetic energy, given the fact that the velocity field employed is the volume-average velocity. In addition, the PFV model does not satisfy the linear momentum balance law that is supposedly formulated using the mass-average velocity [55]. These discrepancies between the models perhaps contribute to the difference in the model predictions in the numerical experiments that we show in the paper.

The remainder of this paper is organized as follows: in section 2, we introduce some notations to simplify the subsequent presentation. In sections 3 and 4 , both a traditional structure-preserving algorithm and an energy quadratization method are developed for the quasi-incompressible hydrodynamic model and the incompressible hydrodynamic model, respectively. Section 5 is devoted to the numerical test of the linear schemes and to show some numerical examples involving drop dynamics and coarsening dynamics. Finally, we finish the paper with a concluding remark in section 6 .

2. Notations. For immiscible binary fluids, one choice of the bulk energy density is given by a double-well repulsive potential,

$$
f(\phi)=\gamma_{2} \phi^{2}(1-\phi)^{2}
$$

where $\gamma_{2}$ measures the strength of the repulsive potential. In fact, the Cahn-Hilliard equation using double-well bulk potential cannot ensure $\phi \in[0,1]$. In order to control numerically $\phi \in(-\epsilon, 1+\epsilon)$ for a small positive number $\epsilon$, we propose a second-order smooth modified bulk energy density as

$$
f(\phi)=\gamma_{2}\left(\phi^{2}(1-\phi)^{2}+f_{1}(\phi)\right)
$$

where

$$
f_{1}(\phi)= \begin{cases}0 & \phi \in[0,1] \\ N \phi^{4}(1-\phi)^{4} & \text { otherwise }\end{cases}
$$

Copyright (C) by SIAM. Unauthorized reproduction of this article is prohibited. 
with $N$ a positive parameter. When $N=0$, the modified potential (2.2) reduces to the double-well potential given in (2.1).

For miscible binary polymeric blends, $f(\phi)$ is given by the Flory-Huggins free energy density

$$
f(\phi)=\gamma_{2}\left(\frac{\phi}{N_{1}} \ln \phi+\frac{(1-\phi)}{N_{2}} \ln (1-\phi)+\chi \phi(1-\phi)\right),
$$

where $N_{1}$ and $N_{2}$ are the polymerization indices for phase $A$ and phase $B$, respectively, $\chi$ is the mixing parameter, and $\gamma_{2}$ measures the strength of the bulk potential.

We define the inner product $(\cdot, \cdot)$ and norm $\|\cdot\|$ for functions as follows:

$$
(f, g)=\int_{\Omega} f g d \mathbf{x}, \quad(\mathbf{F}, \mathbf{G})=\sum_{m, n} \int_{\Omega} \mathbf{F}_{m, n} \mathbf{G}_{m, n} d \mathbf{x}, \quad\|\mathbf{F}\|=(\mathbf{F}, \mathbf{F})^{\frac{1}{2}},
$$

where $\Omega=\left[0, L_{x}\right] \times\left[0, L_{y}\right]$ is the domain of the fluid flow, $f$ and $g$ are scalar functions, and $\mathbf{F}$ and $\mathbf{G}$ are second-order tensor functions defined in $\Omega$. With the periodic boundary condition, we have the following integration-by-parts formulas:

$$
\begin{gathered}
\left(f, \partial_{\alpha} g\right)+\left(\partial_{\alpha} f, g\right)=0, \quad \alpha=x \text { or } y, \\
(f, \nabla \cdot \mathbf{v})+(\nabla f, \mathbf{v})=0,
\end{gathered}
$$

and

$$
(\mathbf{v}, \nabla \cdot \mathbf{F})+(\nabla \mathbf{v}, \mathbf{F})=0 .
$$

By a simple calculation, it is readily shown that

$$
(\mathbf{u}, \mathbf{F} \cdot \mathbf{v})=(\mathbf{u v}, \mathbf{F}),
$$

where $\mathbf{u}, \mathbf{v}$ are vector-valued functions and $\mathbf{F}$ is a matrix-valued function.

For a positive integer $N_{t}$, we define the time step as $\Delta t=T / N_{t}, t_{n}=n \Delta t, 0 \leq$ $n \leq N_{t}$. For positive integers $N_{x}, N_{y}$, we define the space steps as $h_{x}=L_{x} / N_{x}, h_{y}=$ $L_{y} / N_{y}, x_{j}=j h_{x}, 0 \leq j \leq N_{x}-1, y_{k}=k h_{y}, 0 \leq k \leq N_{y}-1$. To approximate periodic boundary conditions, we let $x_{-1}=-h_{x}, x_{N_{x}}=N_{x} h_{x}, y_{-1}=-h_{y}, y_{N_{y}}=N_{y} h_{y}$. A grid function $f=\left\{f_{j, k} \mid-1 \leq j \leq N_{x},-1 \leq k \leq N_{y}\right\}$ is called periodic if

(x-periodic) $f_{-1, k}=f_{N_{x}-1, k}, f_{0, k}=f_{N_{x}, k} ;\left(y\right.$-periodic) $f_{j,-1}=f_{j, N_{y}-1}, f_{j, 0}=f_{j, N_{y}}$.

Next, we define the following discrete operators:

$$
\begin{aligned}
& \delta_{t}^{+} f^{n}=\frac{f^{n+1}-f^{n}}{\Delta t}, \quad f^{n+\frac{1}{2}}=\frac{f^{n+1}+f^{n}}{2}, \bar{f}^{n+\frac{1}{2}}=\frac{1}{2}\left(3 f^{n}-f^{n-1}\right), \\
& \delta_{x}^{+} f_{j, k}=\frac{f_{j+1, k}-f_{j, k}}{h_{x}}, \quad \delta_{x}^{-} f_{j, k}=\frac{f_{j, k}-f_{j-1, k}}{h_{x}}, \quad \delta_{\bar{x}} f_{j, k}=\frac{\delta_{x}^{+}+\delta_{x}^{-}}{2} f_{j, k}, \\
& \delta_{y}^{+} f_{j, k}=\frac{f_{j, k+1}-f_{j, k}}{h_{y}}, \quad \delta_{y}^{-} f_{j, k}=\frac{f_{j, k}-f_{j, k-1}}{h_{y}}, \quad \delta_{\bar{y}} f_{j, k}=\frac{\delta_{y}^{+}+\delta_{y}^{-}}{2} f_{j, k}, \\
& \nabla_{h}^{+}=\left(\begin{array}{c}
\delta_{x}^{+} \\
\delta_{y}^{+}
\end{array}\right), \nabla_{h}^{-}=\left(\begin{array}{c}
\delta_{x}^{-} \\
\delta_{y}^{-}
\end{array}\right), \quad \bar{\nabla}_{h}=\frac{\nabla_{h}^{+}+\nabla_{h}^{-}}{2}, \Delta_{h}=\delta_{x}^{+} \delta_{x}^{-}+\delta_{y}^{+} \delta_{y}^{-} .
\end{aligned}
$$

Copyright $@$ by SIAM. Unauthorized reproduction of this article is prohibited. 
The discrete inner product and norm are defined, respectively, for tensor valued functions as follows:

$$
(\mathbf{F}, \mathbf{G})_{h}=\sum_{m, n} \sum_{j=0}^{N_{x}-1} \sum_{k=0}^{N_{y}-1}\left(\mathbf{F}_{m, n}\right)_{j, k}\left(\mathbf{G}_{m, n}\right)_{j, k} h_{x} h_{y}, \quad\|\mathbf{F}\|_{h}=(\mathbf{F}, \mathbf{F})_{h}^{\frac{1}{2}}
$$

The following summation-by-parts formulas are analogous to the integration-by-parts formulas $(2.6)-(2.8)$

$$
\begin{aligned}
& \left(f, \delta_{\alpha}^{-} g\right)_{h}+\left(\delta_{\alpha}^{+} f, g\right)_{h}=0, \\
& \left(f, \delta_{\bar{\alpha}} g\right)_{h}+\left(\delta_{\bar{\alpha}} f, g\right)_{h}=0, \\
& \left(f, \nabla_{h}^{-} \cdot \mathbf{v}\right)_{h}+\left(\nabla_{h}^{+} f, \mathbf{v}\right)_{h}=0, \\
& \left(f, \nabla_{h}^{+} \cdot \mathbf{v}\right)_{h}+\left(\nabla_{h}^{-} f, \mathbf{v}\right)_{h}=0, \\
& \left(f, \bar{\nabla}_{h} \cdot \mathbf{v}\right)_{h}+\left(\bar{\nabla}_{h} f, \mathbf{v}\right)_{h}=0, \\
& \left(\mathbf{v}, \nabla_{h}^{+} \cdot \mathbf{F}\right)_{h}+\left(\nabla_{h}^{-} \mathbf{v}, \mathbf{F}\right)_{h}=0, \\
& \left(\mathbf{v}, \nabla_{h}^{-} \cdot \mathbf{F}\right)_{h}+\left(\nabla_{h}^{+} \mathbf{v}, \mathbf{F}\right)_{h}=0, \\
& \left(\mathbf{v}, \bar{\nabla}_{h} \cdot \mathbf{F}\right)_{h}+\left(\bar{\nabla}_{h} \mathbf{v}, \mathbf{F}\right)_{h}=0,
\end{aligned}
$$

where $\alpha=x$ or $y$. In addition, we have

$$
(\mathbf{u}, \mathbf{F} \cdot \mathbf{v})_{h}=(\mathbf{u v}, \mathbf{F})_{h} .
$$

3. Numerical approximations to the quasi-incompressible model. In this section, we first reformulate the quasi-incompressible model (1.3) into an equivalent form. Then we develop energy stable numerical schemes to solve it.

\subsection{Model reformulation.}

3.1.1. Equivalent system. Multiplying (1.3b) and (1.3c) by $\rho_{2}$ and $\rho_{1}-\rho_{2}$, respectively, then adding the results, we obtain the mass conservation

$$
\partial_{t} \rho+\nabla \cdot(\rho \mathbf{v})=0
$$

Multiplying (3.1) by $\mathbf{v} / 2$, then adding the result to Eq. (1.3a) and using the identity

$$
\nabla \cdot(\rho \mathbf{v v})=\rho \mathbf{v} \cdot \nabla \mathbf{v}+\nabla \cdot(\rho \mathbf{v}) \mathbf{v}
$$

we obtain

$$
\sqrt{\rho} \partial_{t}(\sqrt{\rho} \mathbf{v})+\frac{1}{2}(\rho \mathbf{v} \cdot \nabla \mathbf{v}+\nabla \cdot(\rho \mathbf{v} \mathbf{v}))=-\nabla p+\nabla \cdot \tau-\phi \nabla \mu .
$$

Let $\mathbf{u}=\sqrt{\rho} \mathbf{v}$. Replacing all $\mathbf{v}$ with $\frac{\mathbf{u}}{\sqrt{\rho}}$ in system (1.3), we obtain the following equivalent form:

$$
\begin{aligned}
& \partial_{t} \mathbf{u}+\frac{1}{2}\left(\mathbf{u} \cdot \nabla\left(\frac{\mathbf{u}}{\sqrt{\rho}}\right)+\frac{1}{\sqrt{\rho}} \nabla \cdot(\mathbf{u u})\right)=\frac{1}{\sqrt{\rho}}(-\nabla p+\nabla \cdot \tau-\phi \nabla \mu), \\
& \nabla \cdot\left(\frac{\mathbf{u}}{\sqrt{\rho}}\right)=a \nabla \cdot(\lambda \nabla(\mu+a p)), \\
& \partial_{t} \phi+\nabla \cdot\left(\frac{1}{\sqrt{\rho}} \phi \mathbf{u}\right)=\nabla \cdot(\lambda \nabla(\mu+a p)),
\end{aligned}
$$

Copyright $@$ by SIAM. Unauthorized reproduction of this article is prohibited. 
where the stress tensor is $\tau=2 \eta \mathbf{D}+\nu \operatorname{tr}(\mathbf{D}) \mathbf{I}$ and the strain rate tensor is

$$
\mathbf{D}=\frac{1}{2}\left(\nabla \frac{\mathbf{u}}{\sqrt{\rho}}+\left(\nabla \frac{\mathbf{u}}{\sqrt{\rho}}\right)^{T}\right) .
$$

THEOREM 3.1. With periodic boundary conditions, the solution of system (3.3) satisfies the following energy dissipation law:

$$
\frac{d E}{d t}=-\left(\lambda,|\nabla(\mu+a p)|^{2}\right)-2\left(\eta,|\mathbf{D}|^{2}\right)-\left(\nu,|\operatorname{tr}(\mathbf{D})|^{2}\right),
$$

where $E$ is the total energy of (3.3) defined as

$$
E=\frac{1}{2}\|\mathbf{u}\|^{2}+\frac{\gamma_{1}}{2}\|\nabla \phi\|^{2}+(f(\phi), 1)
$$

Proof. According to (2.8) and (2.9), we have

$$
\left(\mathbf{u}, \mathbf{u} \cdot \nabla\left(\frac{\mathbf{u}}{\sqrt{\rho}}\right)+\frac{1}{\sqrt{\rho}} \nabla \cdot(\mathbf{u u})\right)=0
$$

and

$$
\left(\frac{\mathbf{u}}{\sqrt{\rho}}, \nabla \cdot \tau\right)=-\left(\nabla\left(\frac{\mathbf{u}}{\sqrt{\rho}}\right), \tau\right)=-2\left(\eta,|\mathbf{D}|^{2}\right)-\left(\nu,|\operatorname{tr}(\mathbf{D})|^{2}\right) .
$$

Computing the inner product of (3.3a) with $\mathbf{u}$, then using (3.6), (2.7), and (3.3b), we have

$$
\begin{aligned}
\left(\mathbf{u}, \partial_{t} \mathbf{u}\right) & =\left(\frac{\mathbf{u}}{\sqrt{\rho}},-\nabla p+\nabla \cdot \tau-\phi \nabla \mu\right) \\
& =\left(\nabla \cdot\left(\frac{\mathbf{u}}{\sqrt{\rho}}\right), p\right)+\left(\frac{\mathbf{u}}{\sqrt{\rho}}, \nabla \cdot \tau\right)-\left(\frac{1}{\sqrt{\rho}} \phi \mathbf{u}, \nabla \mu\right) \\
& =-(\lambda \nabla(\mu+a p), a \nabla p)+\left(\frac{\mathbf{u}}{\sqrt{\rho}}, \nabla \cdot \tau\right)+\left(\nabla \cdot\left(\frac{1}{\sqrt{\rho}} \phi \mathbf{u}\right), \mu\right) .
\end{aligned}
$$

Computing the inner product of (3.3c) with $\mu$, we obtain

$$
\left(\mu, \partial_{t} \phi\right)+\left(\mu, \nabla \cdot\left(\frac{1}{\sqrt{\rho}} \phi \mathbf{u}\right)\right)=-(\lambda \nabla(\mu+a p), \nabla \mu),
$$

where (2.7) is used. Adding (3.8) and (3.9) gives

$$
\left(\mathbf{u}, \partial_{t} \mathbf{u}\right)+\left(\mu, \partial_{t} \phi\right)=-\left(\lambda,|\nabla(\mu+a p)|^{2}\right)+\left(\frac{\mathbf{u}}{\sqrt{\rho}}, \nabla \cdot \tau\right) .
$$

Combining (3.7) and (3.10), we have

$$
\begin{aligned}
\frac{d E}{d t} & =\left(\mathbf{u}, \partial_{t} \mathbf{u}\right)+\gamma_{1}\left(\nabla \phi, \nabla \phi_{t}\right)+\left(f^{\prime}(\phi), \phi_{t}\right) \\
& =\left(\mathbf{u}, \partial_{t} \mathbf{u}\right)+\left(\mu, \phi_{t}\right) \\
& =-\left(\lambda,|\nabla(\mu+a p)|^{2}\right)-2\left(\eta,|\mathbf{D}|^{2}\right)-\left(\nu,|\operatorname{tr}(\mathbf{D})|^{2}\right)
\end{aligned}
$$

which leads to (3.4). 
Remark 3.1. The theorem also holds for the following boundary conditions:

$$
\left.\mathbf{u}\right|_{\partial \Omega}=0,\left.\quad \nabla \phi \cdot \mathbf{n}\right|_{\partial \Omega}=0,\left.\quad \nabla(\mu+a p) \cdot \mathbf{n}\right|_{\partial \Omega}=0 .
$$

Throughout this paper, the results are proved with the periodic boundary conditions; but the results involving PDEs and semidiscrete PDEs are valid equally well with (3.11).

3.1.2. Equivalent system based on energy quadratization. We reformulate the model further into an equivalent model with a quadratic energy functional by introducing a new variable $q=\sqrt{f(\phi)}$ where we assume $f>0$;

$$
\begin{aligned}
& \partial_{t} \mathbf{u}+\frac{1}{2}\left(\mathbf{u} \cdot \nabla\left(\frac{\mathbf{u}}{\sqrt{\rho}}\right)+\frac{1}{\sqrt{\rho}} \nabla \cdot(\mathbf{u u})\right)=\frac{1}{\sqrt{\rho}}(-\nabla p+\nabla \cdot \tau-\phi \nabla \mu), \\
& \nabla \cdot\left(\frac{\mathbf{u}}{\sqrt{\rho}}\right)=a \nabla \cdot(\lambda \nabla(\mu+a p)), \\
& \partial_{t} \phi+\nabla \cdot\left(\frac{1}{\sqrt{\rho}} \phi \mathbf{u}\right)=\nabla \cdot(\lambda \nabla(\mu+a p)), \\
& \partial_{t} q=g(\phi) \partial_{t} \phi
\end{aligned}
$$

where the chemical potential $\mu$ is reexpressed as $\mu=2 q g(\phi)-\gamma_{1} \Delta \phi, g(\phi)=\frac{f^{\prime}(\phi)}{2 \sqrt{f(\phi)}}$.

Remark 3.2. For a free energy density $f(\phi)$ bounded below, we can replace $f(\phi)$ with $f(\phi)+C_{0}$, where $C_{0}$ is a large enough positive number such that $f(\phi)+C_{0}>0$ without affecting dynamics of the system. For example, for the Flory-Huggins free energy, we take $C_{0}=\gamma_{2}\left(\frac{1}{N_{1}}+\frac{1}{N_{2}}\right)$ and modify the free energy density (2.4) as follows:

$$
f(\phi)=\gamma_{2}\left(\frac{\phi}{N_{1}} \ln \phi+\frac{(1-\phi)}{N_{2}} \ln (1-\phi)+\chi \phi(1-\phi)\right)+C_{0} .
$$

For $\phi \in(0,1)$ and $N_{1}, N_{2}, \gamma_{2}, \chi>0$, it is readily seen that the modified free energy density (3.13) satisfies $f(\phi)>0$.

Remark 3.3. The choice of the intermediate variable $q$ is not unique. For example, for the free energy density (2.1), we can take $q=\sqrt{\gamma_{2}} \phi(1-\phi)$ and then $g(\phi)=$ $\sqrt{\gamma_{2}}(1-2 \phi)$. For more complicated free energy functionals, we may need to introduce additional new intermediate variables.

THEOREM 3.2. With periodic boundary conditions, the solution of system (3.12) satisfies the energy dissipation law (3.4), where the energy of (3.12) is defined as

$$
E=\frac{1}{2}\|\mathbf{u}\|^{2}+\frac{\gamma_{1}}{2}\|\nabla \phi\|^{2}+\|q\|^{2} .
$$

Proof. The proof is straightforward and is thus omitted.

3.2. Numerical approximations. In this section, we present fully discrete second-order numerical schemes to solve PDEs (3.3) and (3.12).

3.2.1. A nonlinear energy stable scheme. Applying the central difference scheme in space and the Crank-Nicolson-type method in time to system (3.3), we obtain 


$$
\delta_{t}^{+} \mathbf{u}_{j, k}^{n}+\frac{1}{2}\left(\mathbf{u}_{j, k}^{n+\frac{1}{2}} \cdot \bar{\nabla}_{h}\left(\left(\frac{1}{\sqrt{\rho}}\right)_{j, k}^{n+\frac{1}{2}} \mathbf{u}_{j, k}^{n+\frac{1}{2}}\right)+\left(\frac{1}{\sqrt{\rho}}\right)_{j, k}^{n+\frac{1}{2}} \bar{\nabla}_{h} \cdot\left(\mathbf{u}_{j, k}^{n+\frac{1}{2}} \mathbf{u}_{j, k}^{n+\frac{1}{2}}\right)\right)
$$

$$
=\left(\frac{1}{\sqrt{\rho}}\right)_{j, k}^{n+\frac{1}{2}}\left(-\bar{\nabla}_{h} p_{j, k}^{n+\frac{1}{2}}+\bar{\nabla}_{h} \cdot \tau_{j, k}^{n, n+1}-\phi_{j, k}^{n+\frac{1}{2}} \bar{\nabla}_{h} \mu_{j, k}^{n, n+1}\right),
$$

$$
\bar{\nabla}_{h} \cdot\left(\left(\frac{1}{\sqrt{\rho}}\right)_{j, k}^{n+\frac{1}{2}} \mathbf{u}_{j, k}^{n+\frac{1}{2}}\right)=a \bar{\nabla}_{h} \cdot\left(\lambda_{j, k}^{n+\frac{1}{2}} \bar{\nabla}_{h}\left(\mu_{j, k}^{n, n+1}+a p_{j, k}^{n+\frac{1}{2}}\right)\right),
$$

$$
\delta_{t}^{+} \phi_{j, k}^{n}+\bar{\nabla}_{h} \cdot\left(\left(\frac{1}{\sqrt{\rho}}\right)_{j, k}^{n+\frac{1}{2}} \phi_{j, k}^{n+\frac{1}{2}} \mathbf{u}_{j, k}^{n+\frac{1}{2}}\right)=\bar{\nabla}_{h} \cdot\left(\lambda_{j, k}^{n+\frac{1}{2}} \bar{\nabla}_{h}\left(\mu_{j, k}^{n, n+1}+a p_{j, k}^{n+\frac{1}{2}}\right)\right),
$$

where

$$
\begin{aligned}
& \mathbf{D}_{j, k}^{n, n+1}=\frac{1}{2}\left(\bar{\nabla}_{h}\left(\left(\frac{1}{\sqrt{\rho}}\right)_{j, k}^{n+\frac{1}{2}} \mathbf{u}_{j, k}^{n+\frac{1}{2}}\right)+\bar{\nabla}_{h}\left(\left(\frac{1}{\sqrt{\rho}}\right)_{j, k}^{n+\frac{1}{2}} \mathbf{u}_{j, k}^{n+\frac{1}{2}}\right)^{T}\right), \\
& \tau_{j, k}^{n, n+1}=2 \eta_{j, k}^{n+\frac{1}{2}} \mathbf{D}_{j, k}^{n, n+1}+\nu_{j, k}^{n+\frac{1}{2}} \operatorname{tr}\left(\mathbf{D}_{j, k}^{n, n+1}\right) \mathbf{I}, \\
& \mu_{j, k}^{n, n+1}=\frac{\delta f}{\delta\left(\phi_{j, k}^{n+1}, \phi_{j, k}^{n}\right)}-\gamma_{1} \Delta_{h} \phi_{j, k}^{n+\frac{1}{2}}, \quad \frac{\delta f}{\delta(a, b)}= \begin{cases}\frac{f(a)-f(b)}{a-b} & \text { if } a \neq b, \\
f^{\prime}(a) & \text { if } a=b,\end{cases}
\end{aligned}
$$

and $j=0,1, \ldots, N_{x}-1, k=0,1, \ldots, N_{y}-1, n=0,1, \ldots, N_{t}-1$.

THEOREM 3.3. Under the assumption of the periodic boundary condition, scheme (3.15) preserves the discrete energy dissipation law

$\delta_{t}^{+} E^{n}+\left(\lambda^{n+\frac{1}{2}},\left|\bar{\nabla}_{h}\left(\mu^{n, n+1}+a p^{n+\frac{1}{2}}\right)\right|^{2}\right)_{h}+2\left(\eta^{n+\frac{1}{2}},\left|\mathbf{D}^{n, n+1}\right|^{2}\right)_{h}$

$$
+\left(\nu^{n+\frac{1}{2}},\left|\operatorname{tr}\left(\mathbf{D}^{n, n+1}\right)\right|^{2}\right)_{h}=0,
$$

where $E^{n}$ is the discrete energy defined as

$$
E^{n}=\frac{1}{2}\left\|\mathbf{u}^{n}\right\|_{h}^{2}+\frac{\gamma_{1}}{2}\left\|\nabla_{h}^{+} \phi^{n}\right\|_{h}^{2}+\left(f\left(\phi^{n}\right), 1\right)_{h} .
$$

Proof. According to (2.17) and (2.18), we have

$$
\left(\mathbf{u}^{n+\frac{1}{2}}, \mathbf{u}^{n+\frac{1}{2}} \cdot \bar{\nabla}_{h}\left(\left(\frac{1}{\sqrt{\rho}}\right)^{n+\frac{1}{2}} \mathbf{u}^{n+\frac{1}{2}}\right)+\left(\frac{1}{\sqrt{\rho}}\right)^{n+\frac{1}{2}} \bar{\nabla}_{h} \cdot\left(\mathbf{u}^{n+\frac{1}{2}} \mathbf{u}^{n+\frac{1}{2}}\right)\right)_{h}=0
$$

and

$$
\left(\left(\frac{1}{\sqrt{\rho}}\right)^{n+\frac{1}{2}} \mathbf{u}^{n+\frac{1}{2}}, \bar{\nabla}_{h} \cdot \tau^{n, n+1}\right)_{h}=-2\left(\eta^{n+\frac{1}{2}},\left|\mathbf{D}^{n, n+1}\right|^{2}\right)_{h}-\left(\nu^{n+\frac{1}{2}},\left|\operatorname{tr}\left(\mathbf{D}^{n, n+1}\right)\right|^{2}\right)_{h} .
$$

Computing the discrete inner product of (3.15a) with $\mathbf{u}^{n+\frac{1}{2}}$, then using (3.17), (2.14), and (3.15b), we deduce

Copyright (c) by SIAM. Unauthorized reproduction of this article is prohibited. 


$$
\begin{aligned}
\left(\mathbf{u}^{n+\frac{1}{2}}, \delta_{t}^{+} \mathbf{u}^{n}\right)_{h}= & -\left(\lambda^{n+\frac{1}{2}} \bar{\nabla}_{h}\left(\mu^{n, n+1}+a p^{n+\frac{1}{2}}\right), a \bar{\nabla}_{h} p^{n+\frac{1}{2}}\right)_{h} \\
& +\left(\left(\frac{1}{\sqrt{\rho}}\right)^{n+\frac{1}{2}} \mathbf{u}^{n+\frac{1}{2}}, \bar{\nabla}_{h} \cdot \tau^{n, n+1}\right)_{h} \\
& +\left(\bar{\nabla}_{h} \cdot\left(\left(\frac{1}{\sqrt{\rho}}\right)^{n+\frac{1}{2}} \phi^{n+\frac{1}{2}} \mathbf{u}^{n+\frac{1}{2}}\right), \mu^{n, n+1}\right)_{h} .
\end{aligned}
$$

Computing the discrete inner product of (3.15c) with $\mu^{n, n+1}$, we obtain

$$
\begin{gathered}
\left(\mu^{n, n+1}, \delta_{t}^{+} \phi^{n}\right)_{h}+\left(\mu^{n, n+1}, \bar{\nabla}_{h} \cdot\left(\left(\frac{1}{\sqrt{\rho}}\right)^{n+\frac{1}{2}} \phi^{n+\frac{1}{2}} \mathbf{u}^{n+\frac{1}{2}}\right)\right)_{h} \\
=-\left(\lambda^{n+\frac{1}{2}} \bar{\nabla}_{h}\left(\mu^{n, n+1}+a p^{n+\frac{1}{2}}\right), \bar{\nabla}_{h} \mu^{n, n+1}\right)_{h},
\end{gathered}
$$

where (2.14) was used. Adding (3.19) and (3.20) leads to

$$
\begin{aligned}
\left(\mathbf{u}^{n+\frac{1}{2}}, \delta_{t}^{+} \mathbf{u}^{n}\right)_{h}+\left(\mu^{n, n+1}, \delta_{t}^{+} \phi^{n}\right)_{h}= & -\left(\lambda^{n+\frac{1}{2}},\left|\bar{\nabla}_{h}\left(\mu^{n, n+1}+a p^{n+\frac{1}{2}}\right)\right|^{2}\right)_{h} \\
& +\left(\left(\frac{1}{\sqrt{\rho}}\right)^{n+\frac{1}{2}} \mathbf{u}^{n+\frac{1}{2}}, \bar{\nabla}_{h} \cdot \tau^{n, n+1}\right)_{h} .
\end{aligned}
$$

Using (2.12), (3.18), (3.21), and the identity

$$
\delta_{t}^{+}\left(\mathbf{u}^{n} \cdot \mathbf{v}^{n}\right)=\delta_{t}^{+} \mathbf{u}^{n} \cdot \mathbf{v}^{n+\frac{1}{2}}+\mathbf{u}^{n+\frac{1}{2}} \cdot \delta_{t}^{+} \mathbf{v}^{n},
$$

we have

$$
\begin{aligned}
\delta_{t}^{+} E^{n}= & \left(\mathbf{u}^{n+\frac{1}{2}}, \delta_{t}^{+} \mathbf{u}^{n}\right)_{h}+\gamma_{1}\left(\nabla_{h}^{+} \phi^{n+\frac{1}{2}}, \nabla_{h}^{+} \delta_{t}^{+} \phi^{n}\right)_{h}+\left(\delta_{t}^{+} f\left(\phi^{n}\right), 1\right)_{h} \\
= & \left(\mathbf{u}^{n+\frac{1}{2}}, \delta_{t}^{+} \mathbf{u}^{n}\right)_{h}-\gamma_{1}\left(\Delta_{h} \phi^{n+\frac{1}{2}}, \delta_{t}^{+} \phi^{n}\right)_{h}+\left(\frac{\delta f}{\delta\left(\phi^{n+1}, \phi^{n}\right)}, \delta_{t}^{+} \phi^{n}\right)_{h} \\
= & \left(\mathbf{u}^{n+\frac{1}{2}}, \delta_{t}^{+} \mathbf{u}^{n}\right)_{h}+\left(\mu^{n, n+1}, \delta_{t}^{+} \phi^{n}\right)_{h} \\
= & -\left(\lambda^{n+\frac{1}{2}},\left|\bar{\nabla}_{h}\left(\mu^{n, n+1}+a p^{n+\frac{1}{2}}\right)\right|^{2}\right)_{h}-2\left(\eta^{n+\frac{1}{2}},\left|\mathbf{D}^{n, n+1}\right|^{2}\right)_{h} \\
& -\left(\nu^{n+\frac{1}{2}},\left|\operatorname{tr}\left(\mathbf{D}^{n, n+1}\right)\right|^{2}\right)_{h} .
\end{aligned}
$$

Remark 3.4. If only the time discretization is carried out, it can be readily shown that the semidiscrete scheme is energy stable even for the physical boundary condition.

Remark 3.5. In scheme (3.15), the mass-average velocity is computed by

$$
\mathbf{v}_{j, k}^{n}=\frac{\mathbf{u}_{j, k}^{n}}{\sqrt{\rho_{j, k}^{n}}} .
$$

Remark 3.6. Multiplying (3.15b) and (3.15c) by $\rho_{2}$ and $\rho_{1}-\rho_{2}$, respectively, then adding the results, we obtain the discrete mass conservation law:

$$
\delta_{t}^{+} \rho_{j, k}^{n}+\bar{\nabla}_{h} \cdot\left(\rho_{j, k}^{n+\frac{1}{2}}\left(\frac{1}{\sqrt{\rho}}\right)_{j, k}^{n+\frac{1}{2}} \mathbf{u}_{j, k}^{n+\frac{1}{2}}\right)=0 .
$$

Remark 3.7. The time discretization in this scheme is the analogue of the energy dissipation preserving time discretization used in [30].

Copyright $@$ by SIAM. Unauthorized reproduction of this article is prohibited. 
Scheme (3.15) is unconditionally energy stable. However, it is fully implicit and nonlinear. To solve it, a nonlinear iteration scheme has to be designed, which may destroy the unconditional stability, leaving a constraint on the time step. In the following, we revise it to develop a linear, energy stable method based on the energy quadratization technique.

3.2.2. A linear, energy stable scheme. Applying the central difference scheme in space and the linear-implicit Crank-Nicolson-type method in time to system (3.12), we obtain

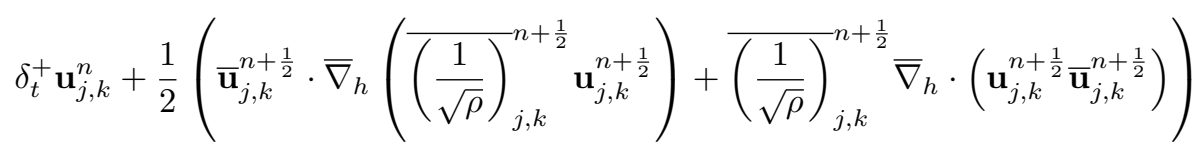

$$
=\left(\frac{1}{\sqrt{\rho}}\right)_{j, k}^{n+\frac{1}{2}}\left(-\bar{\nabla}_{h} p_{j, k}^{n+\frac{1}{2}}+\bar{\nabla}_{h} \cdot \tau_{j, k}^{n, n+1}-\bar{\phi}_{j, k}^{n+\frac{1}{2}} \bar{\nabla}_{h} \mu_{j, k}^{n, n+1}\right),
$$

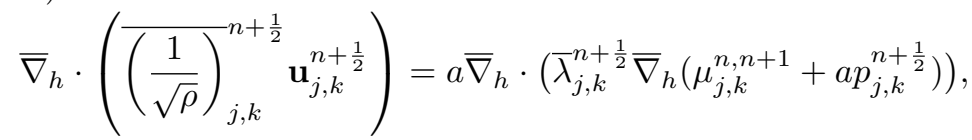

$$
\delta_{t}^{+} \phi_{j, k}^{n}+\bar{\nabla}_{h} \cdot\left(\overline{\left(\frac{1}{\sqrt{\rho}}\right)_{j, k}^{n+\frac{1}{2}}} \bar{\phi}_{j, k}^{n+\frac{1}{2}} \mathbf{u}_{j, k}^{n+\frac{1}{2}}\right)=\bar{\nabla}_{h} \cdot\left(\bar{\lambda}_{j, k}^{n+\frac{1}{2}} \bar{\nabla}_{h}\left(\mu_{j, k}^{n, n+1}+a p_{j, k}^{n+\frac{1}{2}}\right)\right),
$$

$$
\delta_{t}^{+} q_{j, k}^{n}=\overline{g(\phi)}_{j, k}^{n+\frac{1}{2}} \delta_{t}^{+} \phi_{j, k}^{n}
$$

where

$$
\begin{aligned}
\mathbf{D}_{j, k}^{n, n+1} & =\frac{1}{2}\left(\bar{\nabla}_{h}\left(\overline{\left(\frac{1}{\sqrt{\rho}}\right)_{j, k}^{n+\frac{1}{2}}} \mathbf{u}_{j, k}^{n+\frac{1}{2}}\right)+\bar{\nabla}_{h}\left(\overline{\left(\frac{1}{\sqrt{\rho}}\right)_{j, k}^{n+\frac{1}{2}}} \mathbf{u}_{j, k}^{n+\frac{1}{2}}\right)^{T}\right), \\
\tau_{j, k}^{n, n+1} & =2 \bar{\eta}_{j, k}^{n+\frac{1}{2}} \mathbf{D}_{j, k}^{n, n+1}+\bar{\nu}_{j, k}^{n+\frac{1}{2}} \operatorname{tr}\left(\mathbf{D}_{j, k}^{n, n+1}\right) \mathbf{I}, \\
\mu_{j, k}^{n, n+1} & \left.=2 q_{j, k}^{n+\frac{1}{2}} \frac{{ }^{n}}{g(\phi)}\right)_{j, k}^{n+\frac{1}{2}}-\gamma_{1} \Delta_{h} \phi_{j, k}^{n+\frac{1}{2}}
\end{aligned}
$$

and $j=0,1, \ldots, N_{x}-1, k=0,1, \ldots, N_{y}-1, n=1, \ldots, N_{t}-1$.

TheOREm 3.4. Scheme (3.24) preserves the discrete energy dissipation law

$$
\begin{aligned}
& \delta_{t}^{+} E^{n}+\left(\bar{\lambda}^{n+\frac{1}{2}},\left|\bar{\nabla}_{h}\left(\mu^{n, n+1}+a p^{n+\frac{1}{2}}\right)\right|^{2}\right)_{h}+2\left(\bar{\eta}^{n+\frac{1}{2}},\left|\mathbf{D}^{n, n+1}\right|^{2}\right)_{h} \\
& \begin{array}{ll}
(3.25) & +\left(\bar{\nu}^{n+\frac{1}{2}},\left|\operatorname{tr}\left(\mathbf{D}^{n, n+1}\right)\right|^{2}\right)_{h}=0,
\end{array}
\end{aligned}
$$

where the discrete energy is defined as

$$
E^{n}=\frac{1}{2}\left\|\mathbf{u}^{n}\right\|_{h}^{2}+\frac{\gamma_{1}}{2}\left\|\nabla_{h}^{+} \phi^{n}\right\|_{h}^{2}+\left\|q^{n}\right\|_{h}^{2}
$$

Copyright $@$ by SIAM. Unauthorized reproduction of this article is prohibited. 
Proof. Analogous to the proof of Theorem 3.3, we have

$$
\begin{aligned}
\left(\mathbf{u}^{n+\frac{1}{2}}, \delta_{t}^{+} \mathbf{u}^{n}\right)_{h}+\left(\mu^{n, n+1}, \delta_{t}^{+} \phi^{n}\right)_{h}= & -\left(\bar{\lambda}^{n+\frac{1}{2}},\left|\bar{\nabla}_{h}\left(\mu^{n, n+1}+a p^{n+\frac{1}{2}}\right)\right|^{2}\right)_{h} \\
& -2\left(\bar{\eta}^{n+\frac{1}{2}},\left|\mathbf{D}^{n, n+1}\right|^{2}\right)_{h}-\left(\bar{\nu}^{n+\frac{1}{2}},\left|\operatorname{tr}\left(\mathbf{D}^{n, n+1}\right)\right|^{2}\right)_{h} .
\end{aligned}
$$

By a direct calculation, we have

$$
\begin{aligned}
\delta_{t}^{+} E^{n}= & \left(\mathbf{u}^{n+\frac{1}{2}}, \delta_{t}^{+} \mathbf{u}^{n}\right)_{h}+\gamma_{1}\left(\nabla_{h}^{+} \phi^{n+\frac{1}{2}}, \nabla_{h}^{+} \delta_{t}^{+} \phi^{n}\right)_{h}+\left(2 q^{n+\frac{1}{2}}, \delta_{t}^{+} q^{n}\right)_{h} \\
= & \left.\left(\mathbf{u}^{n+\frac{1}{2}}, \delta_{t}^{+} \mathbf{u}^{n}\right)_{h}-\gamma_{1}\left(\Delta_{h} \phi^{n+\frac{1}{2}}, \delta_{t}^{+} \phi^{n}\right)_{h}+\left(2 q^{n+\frac{1}{2}} \overline{g(\phi)}\right)^{n+\frac{1}{2}}, \delta_{t}^{+} \phi^{n}\right)_{h} \\
= & \left(\mathbf{u}^{n+\frac{1}{2}}, \delta_{t}^{+} \mathbf{u}^{n}\right)_{h}+\left(\mu^{n, n+1}, \delta_{t}^{+} \phi^{n}\right)_{h} \\
= & -\left(\bar{\lambda}^{n+\frac{1}{2}},\left|\bar{\nabla}_{h}\left(\mu^{n, n+1}+a p^{n+\frac{1}{2}}\right)\right|^{2}\right)_{h}-2\left(\bar{\eta}^{n+\frac{1}{2}},\left|\mathbf{D}^{n, n+1}\right|^{2}\right)_{h} \\
& -\left(\bar{\nu}^{n+\frac{1}{2}},\left|\operatorname{tr}\left(\mathbf{D}^{n, n+1}\right)\right|^{2}\right)_{h} .
\end{aligned}
$$

This completes the proof.

Remark 3.8. In scheme (3.24), the mass-average velocity can also be computed by (3.22).

Remark 3.9. The discrete mass conservation law also holds:

$$
\delta_{t}^{+} \rho_{j, k}^{n}+\bar{\nabla}_{h} \cdot\left(\bar{\rho}_{j, k}^{n+\frac{1}{2}}\left(\frac{1}{\sqrt{\rho}}\right)_{j, k}^{n+\frac{1}{2}} \mathbf{u}_{j, k}^{n+\frac{1}{2}}\right)=0 .
$$

Remark 3.10. If we replace all $\overline{(\cdot)}^{n+\frac{1}{2}}$ with $(\cdot)^{n}$ in (3.24), we obtain a two-level energy stable scheme, which is still linear implicit but is of order 1 in time. In the numerical experiments, we use the two-level scheme to compute the initial data for the second level values of the three-level scheme (3.24), which does not affect the overall accuracy of second-order scheme (3.24).

Remark 3.11. The advantage of scheme (3.24) compared with scheme (3.15) is its linearity. However, we remark the energy stability is derived for the energy defined in (3.26), which may lead to complications to obtain an $H^{m}$ estimate for the numerical solution. One remedy is to redefine $q$ such that the bulk energy is represented by $\alpha \phi^{2}+q^{2}$, where $\alpha$ is a positive parameter. This will be explored in our subsequent studies on error estimates.

4. Numerical approximations to the incompressible model. Here, we present the incompressible model and then reformulate it into an equivalent system. Then, we propose numerical approximations for the equivalent system.

\subsection{Model reformulation and its equivalent system.}

4.1.1. Equivalent system. Multiplying (1.4b) and (1.4c) by $\rho_{2}$ and $\rho_{1}-\rho_{2}$, respectively, then adding the results, we obtain

$$
\partial_{t} \rho+\nabla \cdot \mathbf{a}=0
$$

Multiplying (4.1) by $\mathbf{v} / 2$, then adding the result to Eq. (1.4a) and noticing the identity

$$
\nabla \cdot(\mathbf{v a})=\mathbf{a} \cdot \nabla \mathbf{v}+(\nabla \cdot \mathbf{a}) \mathbf{v}
$$

we obtain

$$
\sqrt{\rho} \partial_{t}(\sqrt{\rho} \mathbf{v})+\frac{1}{2}(\mathbf{a} \cdot \nabla \mathbf{v}+\nabla \cdot(\mathbf{v a}))=-\nabla p+\nabla \cdot \tau-\phi \nabla \mu .
$$

Copyright $@$ by SIAM. Unauthorized reproduction of this article is prohibited. 
Let $\mathbf{u}=\sqrt{\rho} \mathbf{v}$. Replacing all $\mathbf{v}$ with $\frac{\mathbf{u}}{\sqrt{\rho}}$ in the system (1.4), we obtain the following equivalent form:

$$
\begin{aligned}
& \partial_{t} \mathbf{u}+\frac{1}{2}\left(\mathbf{b} \cdot \nabla\left(\frac{\mathbf{u}}{\sqrt{\rho}}\right)+\frac{1}{\sqrt{\rho}} \nabla \cdot(\mathbf{u b})\right)=\frac{1}{\sqrt{\rho}}(-\nabla p+\nabla \cdot \tau-\phi \nabla \mu), \\
& \nabla \cdot\left(\frac{\mathbf{u}}{\sqrt{\rho}}\right)=0, \\
& \partial_{t} \phi+\nabla \cdot\left(\frac{1}{\sqrt{\rho}} \phi \mathbf{u}\right)=\nabla \cdot(\lambda \nabla \mu),
\end{aligned}
$$

where $\mathbf{b}=\frac{\mathbf{a}}{\sqrt{\rho}}=\mathbf{u}+\frac{1}{\sqrt{\rho}}\left(\rho_{2}-\rho_{1}\right) \lambda \nabla \mu$.

Remark 4.1. If $\mathbf{b}=\mathbf{u}$ in (4.3a) (or $\mathbf{a}=\rho \mathbf{v}$ in (4.2)), model (4.3) reduces to another phase field model proposed in [44].

THEOREM 4.1. With periodic boundary conditions, the solution of system (4.3) satisfies the following energy dissipation law:

$$
\frac{d E}{d t}+\left(\lambda,|\nabla \mu|^{2}\right)+2\left(\eta,|\mathbf{D}|^{2}\right)=0
$$

where $E$ is the total energy of (4.3) defined as

$$
E=\frac{1}{2}\|\mathbf{u}\|^{2}+\frac{\gamma_{1}}{2}\|\nabla \phi\|^{2}+(f(\phi), 1)
$$

Proof. The proof is similar to that of Theorem 3.1 and is thus omitted.

4.1.2. Energy quadratization reformulation. As in the previous section, we introduce a new variable $q=\sqrt{f(\phi)}$ and rewrite system (4.3) as follows:

$$
\begin{aligned}
& \partial_{t} \mathbf{u}+\frac{1}{2}\left(\mathbf{b} \cdot \nabla\left(\frac{\mathbf{u}}{\sqrt{\rho}}\right)+\frac{1}{\sqrt{\rho}} \nabla \cdot(\mathbf{u b})\right)=\frac{1}{\sqrt{\rho}}(-\nabla p+\nabla \cdot \tau-\phi \nabla \mu), \\
& \nabla \cdot\left(\frac{\mathbf{u}}{\sqrt{\rho}}\right)=0, \\
& \partial_{t} \phi+\nabla \cdot\left(\frac{1}{\sqrt{\rho}} \phi \mathbf{u}\right)=\nabla \cdot(\lambda \nabla \mu), \\
& \partial_{t} q=g(\phi) \partial_{t} \phi,
\end{aligned}
$$

where $\mu=2 q g(\phi)-\gamma_{1} \Delta \phi, g(\phi)=\frac{f^{\prime}(\phi)}{2 \sqrt{f(\phi)}}$.

THEOREM 4.2. With periodic boundary conditions, the solution of system (4.6) satisfies the following energy dissipation law:

$$
\frac{d E}{d t}+\left(\lambda,|\nabla \mu|^{2}\right)+2\left(\eta,|\mathbf{D}|^{2}\right)=0,
$$

where $E$ is the total energy of (4.6) defined as

$$
E=\frac{1}{2}\|\mathbf{u}\|^{2}+\frac{\gamma_{1}}{2}\|\nabla \phi\|^{2}+\|q\|^{2} .
$$

Proof. The proof is similar to that of Theorem 3.2 and is thus omitted.

4.2. Numerical approximations. Here we present two energy stable numerical schemes to solve the incompressible model.

4.2.1. Nonlinear energy stable scheme. Applying the central difference scheme in space and the Crank-Nicolson-type method in time for the system (4.3), we obtain 


$$
\begin{aligned}
& \delta_{t}^{+} \mathbf{u}_{j, k}^{n}+\frac{1}{2}\left(\mathbf{b}_{j, k}^{n+\frac{1}{2}} \cdot \bar{\nabla}_{h}\left(\left(\frac{1}{\sqrt{\rho}}\right)_{j, k}^{n+\frac{1}{2}} \mathbf{u}_{j, k}^{n+\frac{1}{2}}\right)+\left(\frac{1}{\sqrt{\rho}}\right)_{j, k}^{n+\frac{1}{2}} \bar{\nabla}_{h} \cdot\left(\mathbf{u}_{j, k}^{n+\frac{1}{2}} \mathbf{b}_{j, k}^{n+\frac{1}{2}}\right)\right) \\
& \text { (4.9a) }=\left(\frac{1}{\sqrt{\rho}}\right)_{j, k}^{n+\frac{1}{2}}\left(-\bar{\nabla}_{h} p_{j, k}^{n+\frac{1}{2}}+\bar{\nabla}_{h} \cdot \tau_{j, k}^{n, n+1}-\phi_{j, k}^{n+\frac{1}{2}} \bar{\nabla}_{h} \mu_{j, k}^{n, n+1}\right) \text {, } \\
& \text { (4.9b) } \bar{\nabla}_{h} \cdot\left(\left(\frac{1}{\sqrt{\rho}}\right)_{j, k}^{n+\frac{1}{2}} \mathbf{u}_{j, k}^{n+\frac{1}{2}}\right)=0 \\
& \text { (4.9c) } \delta_{t}^{+} \phi_{j, k}^{n}+\bar{\nabla}_{h} \cdot\left(\left(\frac{1}{\sqrt{\rho}}\right)_{j, k}^{n+\frac{1}{2}} \phi_{j, k}^{n+\frac{1}{2}} \mathbf{u}_{j, k}^{n+\frac{1}{2}}\right)=\bar{\nabla}_{h} \cdot\left(\lambda_{j, k}^{n+\frac{1}{2}} \bar{\nabla}_{h} \mu_{j, k}^{n, n+1}\right) \text {, }
\end{aligned}
$$

where

$$
\begin{aligned}
& \mathbf{b}_{j, k}^{n}=\mathbf{u}_{j, k}^{n}+\frac{1}{\sqrt{\rho_{j, k}^{n}}}\left(\rho_{2}-\rho_{1}\right) \lambda_{j, k}^{n} \bar{\nabla}_{h} \mu_{j, k}^{n}, \quad \mu_{j, k}^{n}=f^{\prime}\left(\phi_{j, k}^{n}\right)-\gamma_{1} \Delta_{h} \phi_{j, k}^{n}, \\
& \mathbf{D}_{j, k}^{n, n+1}=\frac{1}{2}\left(\bar{\nabla}_{h}\left(\left(\frac{1}{\sqrt{\rho}}\right)_{j, k}^{n+\frac{1}{2}} \mathbf{u}_{j, k}^{n+\frac{1}{2}}\right)+\bar{\nabla}_{h}\left(\left(\frac{1}{\sqrt{\rho}}\right)_{j, k}^{n+\frac{1}{2}} \mathbf{u}_{j, k}^{n+\frac{1}{2}}\right)^{T}\right), \\
& \tau_{j, k}^{n, n+1}=2 \eta_{j, k}^{n+\frac{1}{2}} \mathbf{D}_{j, k}^{n, n+1}, \quad \mu_{j, k}^{n, n+1}=\frac{\delta f}{\delta\left(\phi_{j, k}^{n+1}, \phi_{j, k}^{n}\right)}-\gamma_{1} \Delta_{h} \phi_{j, k}^{n+\frac{1}{2}},
\end{aligned}
$$

and $j=0,1, \ldots, N_{x}-1, k=0,1, \ldots, N_{y}-1, n=0,1, \ldots, N_{t}-1$.

TheOREm 4.3. The scheme (4.9) preserves the discrete energy dissipation law

$$
\delta_{t}^{+} E^{n}+\left(\lambda^{n+\frac{1}{2}},\left|\bar{\nabla}_{h} \mu^{n, n+1}\right|^{2}\right)_{h}+2\left(\eta^{n+\frac{1}{2}},\left|\mathbf{D}^{n, n+1}\right|^{2}\right)_{h}=0,
$$

where $E^{n}$ is the discrete energy defined as

$$
E^{n}=\frac{1}{2}\left\|\mathbf{u}^{n}\right\|_{h}^{2}+\frac{\gamma_{1}}{2}\left\|\nabla_{h}^{+} \phi^{n}\right\|_{h}^{2}+\left(f\left(\phi^{n}\right), 1\right)_{h} .
$$

Proof. The proof is similar to that of Theorem 3.3 and is thus omitted.

4.2.2. Linear, energy stable scheme. Applying the central difference scheme in space and the linear-implicit Crank-Nicolson-type method in time to system (4.6), we obtain

$$
\begin{aligned}
& \delta_{t}^{+} \mathbf{u}_{j, k}^{n}+\frac{1}{2}\left(\overline{\mathbf{b}}_{j, k}^{n+\frac{1}{2}} \cdot \bar{\nabla}_{h}\left({\overline{\left(\frac{1}{\sqrt{\rho}}\right)_{j, k}^{n+\frac{1}{2}}}}_{\mathbf{u}_{j, k}^{n+\frac{1}{2}}}\right)+{\overline{\left(\frac{1}{\sqrt{\rho}}\right)_{j, k}^{n+\frac{1}{2}}}}_{\left.\nabla_{h} \cdot\left(\mathbf{u}_{j, k}^{n+\frac{1}{2}} \overline{\mathbf{b}}_{j, k}^{n+\frac{1}{2}}\right)\right)}\right.
\end{aligned}
$$

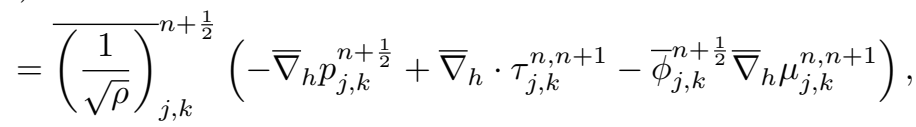

$$
\begin{aligned}
& \bar{\nabla}_{h} \cdot\left(\overline{\left(\frac{1}{\sqrt{\rho}}\right)_{j, k}^{n+\frac{1}{2}}} \mathbf{u}_{j, k}^{n+\frac{1}{2}}\right)=0
\end{aligned}
$$

Copyright $@$ by SIAM. Unauthorized reproduction of this article is prohibited. 


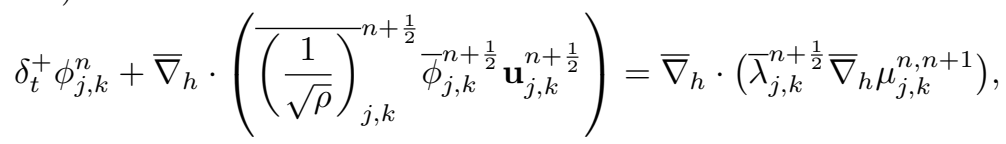

$$
\begin{aligned}
& \delta_{t}^{+} q_{j, k}^{n}=\overline{g(\phi)}_{j, k}^{n+\frac{1}{2}} \delta_{t}^{+} \phi_{j, k}^{n},
\end{aligned}
$$

where

$$
\begin{aligned}
& \mathbf{b}_{j, k}^{n}=\mathbf{u}_{j, k}^{n}+\frac{1}{\sqrt{\rho_{j, k}^{n}}}\left(\rho_{2}-\rho_{1}\right) \lambda_{j, k}^{n} \bar{\nabla}_{h} \mu_{j, k}^{n}, \quad \mu_{j, k}^{n}=2 q_{j, k}^{n} g\left(\phi_{j, k}^{n}\right)-\gamma_{1} \Delta_{h} \phi_{j, k}^{n},
\end{aligned}
$$

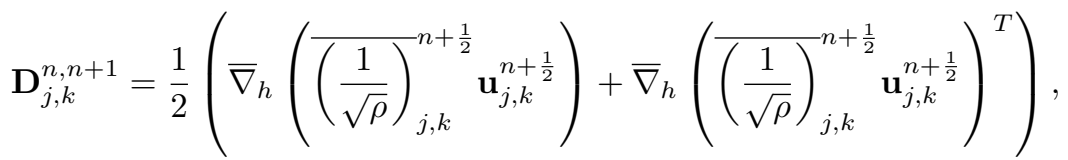

$$
\begin{aligned}
& \tau_{j, k}^{n, n+1}=2 \bar{\eta}_{j, k}^{n+\frac{1}{2}} \mathbf{D}_{j, k}^{n, n+1}, \quad \mu_{j, k}^{n, n+1}=2 q_{j, k}^{n+\frac{1}{2}} \overline{g(\phi)}_{j, k}^{n+\frac{1}{2}}-\gamma_{1} \Delta_{h} \phi_{j, k}^{n+\frac{1}{2}},
\end{aligned}
$$

and $j=0,1, \ldots, N_{x}-1, k=0,1, \ldots, N_{y}-1, n=1, \ldots, N_{t}-1$.

ThEOREM 4.4. Scheme (4.11) preserves the discrete energy dissipation law

$$
\delta_{t}^{+} E^{n}+\left(\bar{\lambda}^{n+\frac{1}{2}},\left|\bar{\nabla}_{h} \mu^{n, n+1}\right|^{2}\right)_{h}+2\left(\bar{\eta}^{n+\frac{1}{2}},\left|\mathbf{D}^{n, n+1}\right|^{2}\right)_{h}=0,
$$

where the discrete energy is defined as

$$
E^{n}=\frac{1}{2}\left\|\mathbf{u}^{n}\right\|_{h}^{2}+\frac{\gamma_{1}}{2}\left\|\nabla_{h}^{+} \phi^{n}\right\|_{h}^{2}+\left\|q^{n}\right\|_{h}^{2}
$$

Proof. The proof is similar to that of Theorem 3.4 and is thus omitted.

Remark 4.2. For scheme (4.11), the volume-average velocity is computed by (3.22).

Remark 4.3. If we replace all $\overline{(\cdot)}^{n+\frac{1}{2}}$ with $(\cdot)^{n+\frac{1}{2}}$ in (4.11), we obtain a new energy stable scheme, which is of second order in time but is nonlinear.

Remark 4.4. If we replace all $\overline{(\cdot)^{n+\frac{1}{2}}}$ with $(\cdot)^{n}$ in $(4.11)$, we obtain a two-level energy stable scheme, which is linear-implicit but of order 1 in time. In the numerical experiments, we use the two-level scheme to compute the initial data for the secondlevel values of the three-level scheme (4.11).

5. Numerical results. In this section, we present several numerical studies to test the accuracy and to show the efficiency of our proposed linear energy stable schemes. In particular, by proposing a specific solution and calculating the numerical errors with respect to different temporal and spatial steps, a second-order spatialtemporal convergence rate is established. The new linear, energy stable schemes are applied to the two models to showcase their differences. Although our proposed numerical schemes don't have any constraints on the choice of the bulk potential, we focus on the double-well potential (2.2) in the studies for simplicity. Readers who are interested in the linearization strategy and related simulations for the Flory-Huggin potential should refer to our work in $[25,26]$.

For periodic boundary conditions, we solve the linear system (3.24) or (4.11) by using a simple fixed-point iteration method similar to the method used in Ref. [24]. In this iteration process, we make the variable coefficient linear terms explicit while 
retaining the implicit constant linear terms. In every iteration step, the linear system is solved by the fast Fourier transform (FFT) algorithm.

Here we take the scheme (3.24) as an example to show the iteration method. For the sake of simplicity, we set $\lambda$ constant and denote $\rho_{m}=\max \left(\rho_{1}, \rho_{2}\right), \eta_{m}=$ $\max \left(\eta_{1}, \eta_{2}\right), \nu_{m}=\max \left(\nu_{1}, \nu_{2}\right)$, and $\mathbf{u}:=\mathbf{u}^{n+\frac{1}{2}}, \overline{\mathbf{u}}:=\overline{\mathbf{u}}^{n+\frac{1}{2}}$, etc. Let $\bar{\psi}=\overline{\frac{1}{\sqrt{\rho}}}$ and $\mathbf{v}=\bar{\psi} \mathbf{u}$, linear scheme (3.24) can be written as

$$
\begin{aligned}
& \bar{\nabla}_{h} p=\eta_{m} \bar{\Delta}_{h} \mathbf{v}+\left(\eta_{m}+\nu_{m}\right) \bar{\nabla}_{h}\left(\bar{\nabla}_{h} \cdot \mathbf{v}\right)+\mathbf{g}, \\
& \bar{\nabla}_{h} \cdot \mathbf{v}=a \lambda \bar{\Delta}_{h} \mu+a^{2} \lambda \bar{\Delta}_{h} p, \\
& \frac{2}{\Delta t}\left(\phi-\phi^{n}\right)+\bar{\nabla}_{h} \cdot(\bar{\phi} \mathbf{v})=\lambda \bar{\Delta}_{h} \mu+a \lambda \bar{\Delta}_{h} p,
\end{aligned}
$$

where the spatial subscripts were omitted for simplicity, $\bar{\Delta}_{h}=\bar{\nabla}_{h} \cdot \bar{\nabla}_{h}$, and

$$
\begin{aligned}
\mathbf{g}(\mathbf{v}, \widetilde{\tau}, \mu) & =\bar{\nabla}_{h} \cdot \widetilde{\tau}-\bar{\phi}_{h} \mu-\frac{2}{\bar{\psi} \Delta t}\left(\frac{\mathbf{v}}{\bar{\psi}}-\mathbf{u}^{n}\right)-\frac{1}{2}\left(\frac{\overline{\mathbf{u}}}{\bar{\psi}} \cdot \bar{\nabla}_{h} \mathbf{v}+\bar{\nabla}_{h} \cdot\left(\frac{\mathbf{v}}{\bar{\psi}} \overline{\mathbf{u}}\right)\right), \\
\widetilde{\tau}(\mathbf{v}) & =\left(\bar{\eta}-\eta_{m}\right)\left(\bar{\nabla}_{h} \mathbf{v}+\bar{\nabla}_{h} \mathbf{v}^{T}\right)+\left(\bar{\nu}-\nu_{m}\right)\left(\bar{\nabla}_{h} \cdot \mathbf{v}\right) \mathbf{I}, \\
\mu(\phi, q) & =2 \overline{g(\phi)} q-\gamma_{1} \Delta_{h} \phi \\
q(\phi) & =q^{n}+\overline{g(\phi)}\left(\phi-\phi^{n}\right) .
\end{aligned}
$$

Note that $\mathbf{g}$ is regarded as a function of $\mathbf{v}, \widetilde{\tau}, \mu$, etc. Taking the discrete divergence on both sides of (5.1a), then using (5.1b), we obtain

$$
\left(1-a^{2} \lambda\left(2 \eta_{m}+\nu_{m}\right) \bar{\Delta}_{h}\right) \bar{\Delta}_{h} p=a \lambda\left(2 \eta_{m}+\nu_{m}\right) \bar{\Delta}_{h}^{2} \mu+\bar{\nabla}_{h} \cdot \mathbf{g} .
$$

Given the initial iteration $\mathbf{v}_{0}=\bar{\psi} \mathbf{u}^{n}$ and $\phi_{0}=\phi^{n}$, we compute $\left(\phi_{s+1}, \mathbf{v}_{s+1}\right)$ for $s \geq 0$. Step 1:

$$
\left\{\begin{aligned}
\left(1-a^{2} \lambda\left(2 \eta_{m}+\nu_{m}\right) \bar{\Delta}_{h}\right) \bar{\Delta}_{h} p_{1}= & a \lambda\left(2 \eta_{m}+\nu_{m}\right) \bar{\Delta}_{h}^{2} \mu\left(\phi_{s+1}, q\left(\phi_{s}\right)\right) \\
& +\bar{\nabla}_{h} \cdot \mathbf{g}\left(\mathbf{v}_{s}, \widetilde{\tau}\left(\mathbf{v}_{s}\right), \mu\left(\phi_{s}, q\left(\phi_{s}\right)\right)\right) \\
\frac{2}{\Delta t}\left(\phi_{s+1}-\phi^{n}\right)+\bar{\nabla}_{h} \cdot\left(\bar{\phi}_{s}\right)= & \lambda \bar{\Delta}_{h} \mu\left(\phi_{s+1}, q\left(\phi_{s}\right)\right)+a \lambda \bar{\Delta}_{h} p_{1}
\end{aligned}\right.
$$

Step 2:

$$
\left\{\begin{array}{c}
\left(1-a^{2} \lambda\left(2 \eta_{m}+\nu_{m}\right) \bar{\Delta}_{h}\right) \bar{\Delta}_{h} p_{2}=a \lambda\left(2 \eta_{m}+\nu_{m}\right) \bar{\Delta}_{h}^{2} \mu\left(\phi_{s+1}, q\left(\phi_{s+1}\right)\right) \\
+\bar{\nabla}_{h} \cdot \mathbf{g}\left(\mathbf{v}_{s}, \widetilde{\tau}\left(\mathbf{v}_{s}\right), \mu\left(\phi_{s+1}, q\left(\phi_{s+1}\right)\right)\right) \\
\frac{2}{\Delta t} \rho_{m} \mathbf{v}_{s+1}+\bar{\nabla}_{h} p_{2}=\eta_{m} \bar{\Delta}_{h} \mathbf{v}_{s+1}+\left(\eta_{m}+\nu_{m}\right) \bar{\nabla}_{h}\left(\bar{\nabla}_{h} \cdot \mathbf{v}_{s+1}\right) \\
+\mathbf{g}\left(\mathbf{v}_{s}, \widetilde{\tau}\left(\mathbf{v}_{s}\right), \mu\left(\phi_{s+1}, q\left(\phi_{s+1}\right)\right)\right)+\frac{2}{\Delta t} \rho_{m} \mathbf{v}_{s}
\end{array}\right.
$$

At step 1, we apply the FFT algorithm to first eliminate $p_{1}$ and then compute $\phi_{s+1}$. Similarly, we calculate $\mathbf{v}_{s+1}$ from step 2. For more details, please refer to [24].

5.1. Mesh refinement test. In order to test the convergence rate of the proposed schemes, we consider the two reformulated models (3.12) and (4.6) with the double-well free energy (2.1) in a rectangular domain $\Omega=[0,2 \pi]^{2}$. We make the following functions exact solution of the system modified by some appropriate forcing functions:

Copyright $@$ by SIAM. Unauthorized reproduction of this article is prohibited. 


$$
\left\{\begin{array}{l}
u_{1}=\sqrt{\rho} \sin (x) \cos (y) \sin (t), \\
u_{2}=\sqrt{\rho} \cos (x) \sin (y) \sin (t), \\
\phi=\cos (x) \cos (y) \cos (t), \\
p=\sin (x) \sin (y) \sin (t), \\
q=\sqrt{\gamma_{2}} \phi(1-\phi),
\end{array}\right.
$$

where $\rho=\left(\rho_{1}-\rho_{2}\right) \phi+\rho_{2}$. Here we note that $g(\phi)=\sqrt{\gamma_{2}}(1-2 \phi)$. The parameter values are chosen as

$$
\rho_{1}=\eta_{1}=\nu_{1}=1, \quad \rho_{2}=\eta_{2}=\nu_{2}=3, \quad \gamma_{1}=0.01, \quad \gamma_{2}=1, \quad \lambda=10^{-4} .
$$

We choose the number of spatial grids as $N_{x}=N_{y}$ and compare the numerical solution with the exact solution at $t=1$. We compute $L_{2}$ and $L_{\infty}$ errors of velocity $v_{1}$, phase variable $\phi$, and new variable $q$ by varying the grid size in space and time. From Tables 1-6, we observe that both linear schemes are second-order accurate in time and space for all variables.

5.2. Comparison of coarsening dynamics between the two models. Next, we study coarsening dynamics using the double-well potential given in (2.2). Here, we use periodic boundary conditions and $N_{x}=N_{y}=129$ mesh points in each direction in space. The initial condition is chosen as

$$
\phi=0.5+0.4 \sin (4 \pi x) \sin (4 \pi y) .
$$

The parameter values are chosen as

$$
\gamma_{1}=10^{-2}, \quad \gamma_{2}=10^{2}, \quad \eta_{1}=\eta_{2}=1, \quad \lambda=10^{-7}, \quad N=10^{7} .
$$

\begin{tabular}{|c|c|c|c|c|c|c|}
\hline \multirow[t]{2}{*}{$\bar{\tau}$} & \multirow[t]{2}{*}{$N$} & \multicolumn{2}{|c|}{ Error } & \multicolumn{2}{|c|}{ Order } & \multirow[t]{2}{*}{$\mathrm{CPU}(\mathrm{s})$} \\
\hline & & $L^{\infty}$ & $L^{2}$ & $L^{\infty}$ & $L^{2}$ & \\
\hline 0.1 & 16 & $2.5114 \mathrm{e}-02$ & $8.1123 \mathrm{e}-02$ & - & - & 0.4 \\
\hline 0.05 & 32 & $5.8939 \mathrm{e}-03$ & $1.8862 \mathrm{e}-02$ & 2.0912 & 2.1046 & 2.6 \\
\hline 0.025 & 64 & $1.4221 \mathrm{e}-03$ & $4.5426 \mathrm{e}-03$ & 2.0512 & 2.0539 & 26.5 \\
\hline 0.0125 & 128 & $3.5139 \mathrm{e}-04$ & $1.1210 \mathrm{e}-03$ & 2.0168 & 2.0187 & 322.4 \\
\hline
\end{tabular}

TABLE 1

Mesh refinement test of scheme (3.24) for $v_{1}$ (or $v_{2}$ ).

\begin{tabular}{|c|c|c|c|c|c|}
\hline \multirow[t]{2}{*}{$\bar{\tau}$} & \multirow[t]{2}{*}{$N$} & \multicolumn{2}{|c|}{ Error } & \multicolumn{2}{|c|}{ Order } \\
\hline & & $L^{\infty}$ & $L^{2}$ & $L^{\infty}$ & $L^{2}$ \\
\hline 0.1 & 16 & $7.0439 \mathrm{e}-02$ & $1.1247 \mathrm{e}-01$ & - & - \\
\hline 0.05 & 32 & $1.9881 \mathrm{e}-02$ & $2.8863 \mathrm{e}-02$ & 1.8250 & 1.9623 \\
\hline 0.025 & 64 & $5.1474 \mathrm{e}-03$ & $7.2673 \mathrm{e}-03$ & 1.9495 & 1.9897 \\
\hline 0.0125 & 128 & $1.2985 \mathrm{e}-03$ & $1.8204 \mathrm{e}-03$ & 1.9870 & 1.9972 \\
\hline
\end{tabular}

TABLE 2

Mesh refinement test of scheme (3.24) for $\phi$.

\begin{tabular}{|c|c|c|c|c|c|}
\hline \multirow[t]{2}{*}{$\bar{\tau}$} & \multirow[t]{2}{*}{$N$} & \multicolumn{2}{|c|}{ Error } & \multicolumn{2}{|c|}{ Order } \\
\hline & & $L^{\infty}$ & $L^{2}$ & $L^{\infty}$ & $L^{2}$ \\
\hline$\overline{0.1}$ & 16 & $1.4655 \mathrm{e}-01$ & $1.7039 \mathrm{e}-01$ & - & - \\
\hline 0.05 & 32 & $4.1967 \mathrm{e}-02$ & $4.5579 \mathrm{e}-02$ & 1.8041 & 1.9024 \\
\hline 0.025 & 64 & $1.0913 \mathrm{e}-02$ & $1.1600 \mathrm{e}-02$ & 1.9432 & 1.9743 \\
\hline 0.0125 & 128 & $2.7560 \mathrm{e}-03$ & $2.9132 \mathrm{e}-03$ & 1.9854 & 1.9934 \\
\hline
\end{tabular}

TABLE 3

Mesh refinement test of scheme (3.24) for $q$.

Copyright (c) by SIAM. Unauthorized reproduction of this article is prohibited. 
TABLE 4

Mesh refinement test of scheme (4.11) for $v_{1}$ (or $v_{2}$ ).

\begin{tabular}{llllllll}
\hline$\tau$ & $N$ & \multicolumn{2}{c}{ Error } & & \multicolumn{2}{c}{ Order } & CPU(s) \\
\cline { 3 - 4 } & & $L^{\infty}$ & $L^{2}$ & & $L^{\infty}$ & $L^{2}$ & \\
\hline 0.1 & 16 & $2.5279 \mathrm{e}-02$ & $8.1334 \mathrm{e}-02$ & & - & - & 0.6 \\
0.05 & 32 & $5.9683 \mathrm{e}-03$ & $1.9013 \mathrm{e}-02$ & & 2.0825 & 2.0969 & 3.8 \\
0.025 & 64 & $1.4476 \mathrm{e}-03$ & $4.6073 \mathrm{e}-03$ & & 2.0436 & 2.0450 & 39.7 \\
0.0125 & 128 & $3.5627 \mathrm{e}-04$ & $1.1335 \mathrm{e}-03$ & & 2.0226 & 2.0232 & 480.7 \\
\hline
\end{tabular}

TABLE 5

Mesh refinement test of scheme (4.11) for $\phi$.

\begin{tabular}{lllllll}
\hline$\tau$ & $N$ & \multicolumn{2}{c}{ Error } & & \multicolumn{2}{c}{ Order } \\
\cline { 3 - 4 } \cline { 6 - 7 } & & $L^{\infty}$ & $L^{2}$ & & $L^{\infty}$ & $L^{2}$ \\
\hline 0.1 & 16 & $7.1873 \mathrm{e}-02$ & $1.1328 \mathrm{e}-01$ & & - \\
0.05 & 32 & $2.0516 \mathrm{e}-02$ & $2.9130 \mathrm{e}-02$ & & 1.8087 & 1.9593 \\
0.025 & 64 & $5.3402 \mathrm{e}-03$ & $7.3405 \mathrm{e}-03$ & & 1.9418 & 1.9885 \\
0.0125 & 128 & $1.3500 \mathrm{e}-03$ & $1.8397 \mathrm{e}-03$ & & 1.9839 & 1.9964 \\
\hline
\end{tabular}

TABLE 6

Mesh refinement test of scheme (4.11) for $q$.

\begin{tabular}{|c|c|c|c|c|c|}
\hline \multirow[t]{2}{*}{$\bar{\tau}$} & \multirow[t]{2}{*}{$N$} & \multicolumn{2}{|c|}{ Error } & \multicolumn{2}{|c|}{ Order } \\
\hline & & $L^{\infty}$ & $L^{2}$ & $L^{\infty}$ & $L^{2}$ \\
\hline$\overline{0.1}$ & 16 & $1.4936 \mathrm{e}-01$ & $1.7230 \mathrm{e}-01$ & - & - \\
\hline 0.05 & 32 & $4.3266 \mathrm{e}-02$ & $4.6246 \mathrm{e}-02$ & 1.7875 & 1.8975 \\
\hline 0.025 & 64 & $1.1312 \mathrm{e}-02$ & $1.1785 \mathrm{e}-02$ & 1.9353 & 1.9723 \\
\hline 0.0125 & 128 & $2.8631 \mathrm{e}-03$ & $2.9620 \mathrm{e}-03$ & 1.9822 & 1.9924 \\
\hline
\end{tabular}

Here, we conduct several numerical experiments by varying the density ratio $\rho_{1}: \rho_{2}$, while keeping the other parameter values unchanged. The simulation results by comparing model (3.12) with model (3.3) are shown in Figure 1. The energy decay curve with time is shown in Figure 2. Some interesting phenomena are observed.

First, in the quasi-incompressible model, when the density ratio is 10:1, fluid phase 1 forms drops within the matrix of the other fluid phase shown in Figure 1(a). The drops form quickly in this simulation such that the total energy decays quickly to a saturated level. Likewise, when the density ratio is reversed to 1:10, the heavy fluid component forms drops within the lighter fluid component shown in 1(b). From the energy curve in Figure 2, we observe that the coarsening dynamics in this case is the slowest. At $t=1000$, the fluid matrix phase has not reached a quasi-steady state. In both cases, the heavy fluid component exists in the form of drops in the matrix of the lighter fluid component. This symmetry in morphology is well exhibited in the figure. When the density ratio is 1 , the PFM model reduces to an incompressible model and the phases are separated into stripe patterns. At the moment when stripes form, the energy drops a step in time. In contrast, we observe that coarsening dynamics predicted by the incompressible model (PFV) is less sensitive to the change of the density ratio as shown in Figures 1(e) and 1(f). Regardless of the density ratio, it's always the second fluid component that forms drops within the matrix of the first fluid component in the two case studies presented. In fact, the corresponding energy curves in Figure 2 are indistinguishable with respect to the two density ratios 1:10 and 10:1. They do not show morphological symmetry given the symmetric initial profile. This result seems to be unphysical, which indicates the model is not physically valid.

In addition, by investigating the energy curves in Figure 2, we observe that there does not exist a linear correlation between dynamics predicted by these two models. 

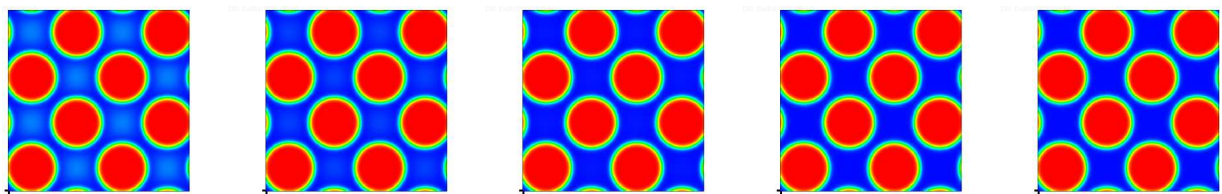

(a) PFM model, $\rho_{1}=10, \rho_{2}=1, t=50,100,200,500,1000$
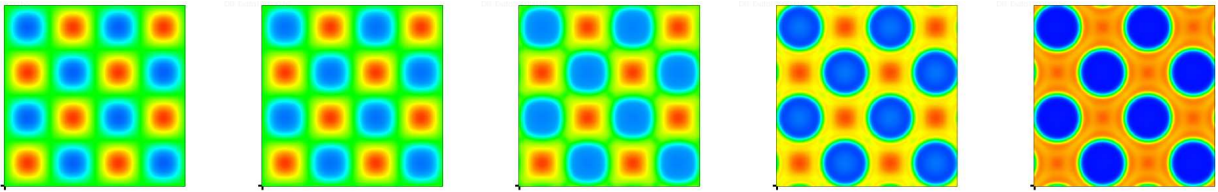

(b) PFM model, $\rho_{1}=1, \rho_{2}=10, t=50,100,200,500,1000$
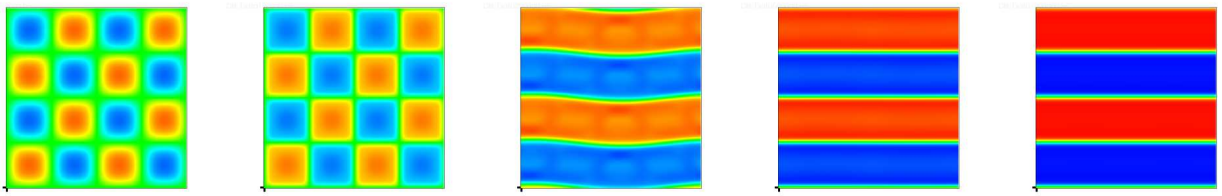

(c) $\rho_{1}=\rho_{2}=1, t=50,100,200,500,1000$
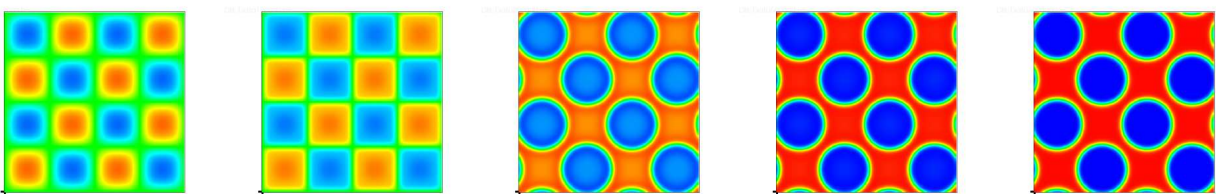

(d) PFV model, $\rho_{1}=1, \rho_{2}=10, t=50,100,200,500,1000$
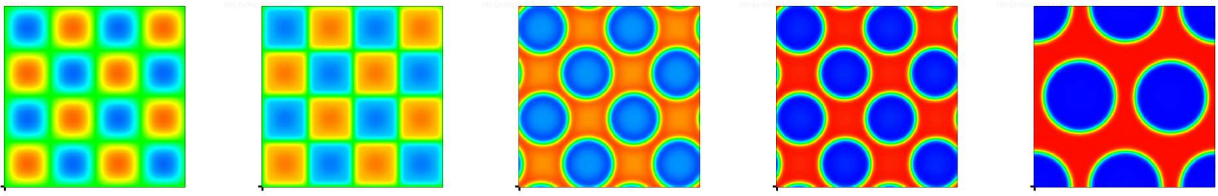

(e) PFV model, $\rho_{1}=10, \rho_{2}=1, t=50,100,200,500,1000$

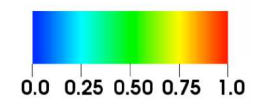

(f) Color Bar

FIG. 1. Coarsening dynamics. In the PFM model, the density ratio has dramatic effects on the coarsening dynamics. The PFV model gives similar dynamics for both fluid components. Its dynamics is less correlated with the density ratio. However, different density does induce the different coarsening rate shown in the energy decay curve in time. Here, we use a standard heat color map to plot the volume fraction of fluid $1, \phi$, where red represents 1 and blue represents 0 in (f).

For the PFM model, the case of $\rho_{1}>\rho_{2}$ seems to predict faster dynamics initially than the case of $\rho_{2}>\rho_{1}$. This in fact can be seen from the effective mobility parameter in transport equations for different phases, which are density ratio dependent in the PFM formulation. In the PFV formulation, mobility parameters in the transport 


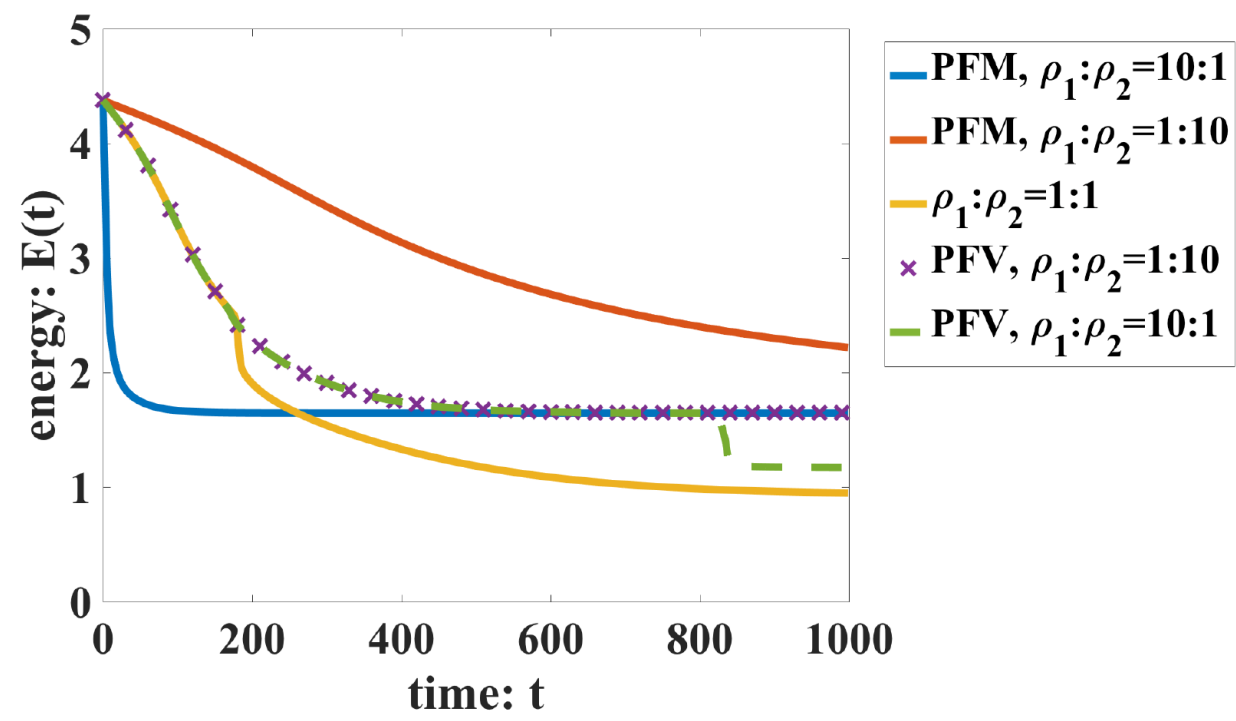

FIG. 2. Energy as a function of time for the coarsening dynamics in Figure 3. Near $t=800$, the PFV model predicts a coarsening transition for density ratio 10:1, indicating the PFV model is also inherently affected weakly by the density difference.

equations for different phases are identical. But, when the density ratio is 10:1 and after $t=800$, the morphology coarsens to form larger drops of fluid 2 within fluid 1 , indicated by the step drop in the energy curve. These simulations show that dynamics predicted by the PFM model is quite different in the beginning for different density ratios, whereas dynamics predicted by the PFV model may be similar initially, but it can have different coarsening transition later on.

Next, we study coarsening dynamics of two drops of fluid 1 immersed in the matrix of fluid 2. Again, the two models predict quite different dynamics. The parameter values used in the numerical study are chosen as

$$
\gamma_{1}=10^{-2}, \quad \gamma_{2}=10^{2}, \quad \eta_{1}=\eta_{2}=1, \quad \lambda=10^{-7} .
$$

The initial condition is given by

$\phi(t=0)=\max \left(\frac{1+\tanh \frac{0.15-R_{1}}{\varepsilon}}{2}, \frac{1+\tanh \frac{0.15-R_{2}}{\varepsilon}}{2}, \frac{1+\tanh \frac{\varepsilon-R_{3}}{\varepsilon}}{2} \frac{\tanh \frac{0.25-R_{4}}{\varepsilon}+1}{2}\right)$,

where

$$
R_{1}=\sqrt{\left(x-0.5 L_{x}\right)^{2}+\left(y-0.25 L_{y}\right)^{2}}, \quad R_{2}=\sqrt{\left(x-0.5 L_{x}\right)^{2}+\left(Y-0.75 L_{y}\right)^{2}},
$$

$$
R_{3}=\left|x-0.5 L_{x}\right|, \quad R_{4}=\left|y-0.5 L_{y}\right|, \quad \varepsilon=0.01, \quad L_{x}=L_{y}=1,
$$

and $\mathbf{v}(t=0)=0$. The initial condition for pressure is not necessary. Here, we use the periodic boundary condition once again.

The two drops initially connected by a thin liquid bridge always break up into separate drops as predicted by the PFM model, as the density difference is large. The results for density ratios 10:1 and 1:10 are depicted in Figures 3(a) and 3(b), respectively. Similar to the case depicted in Figure 1, the PFM model with $\rho_{1} \neq \rho_{2}$ predicts faster dynamics initially. However, it would be trapped in a local minimum, 

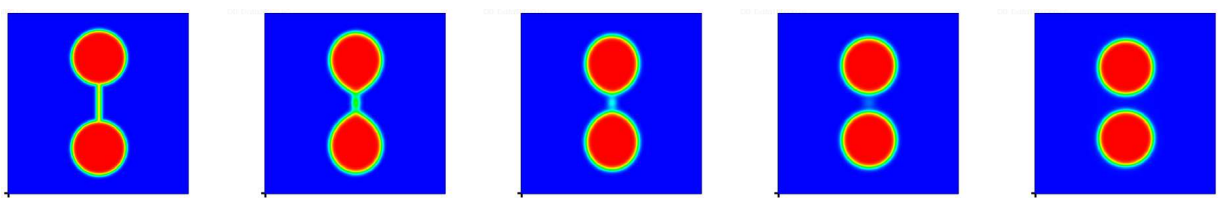

(a) PFM model, $\rho_{1}=10, \rho_{2}=1, t=0,2,5,10,150$
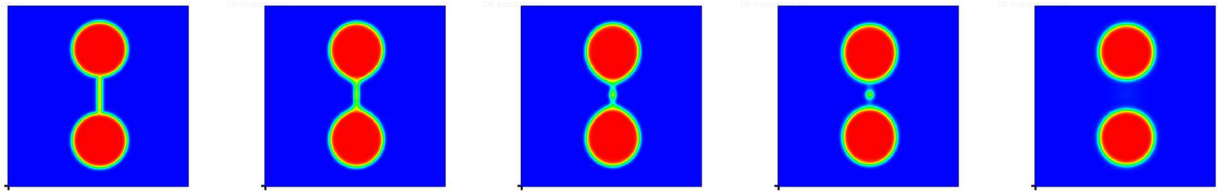

(b) PFM model, $\rho_{1}=1, \rho_{2}=10, t=0,2,5,10,150$
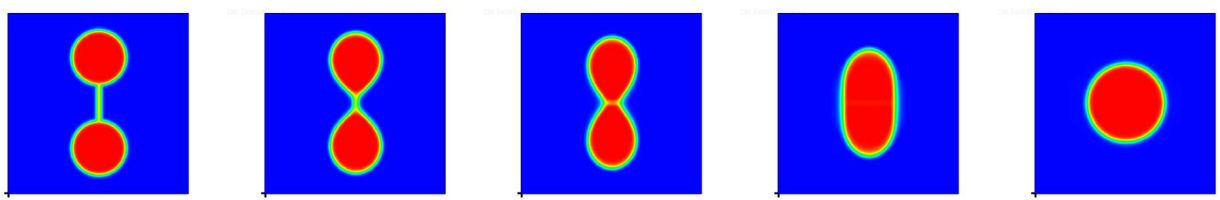

(c) PFM model, $\rho_{1}=1, \rho_{2}=1, t=0,2,5,10,150$
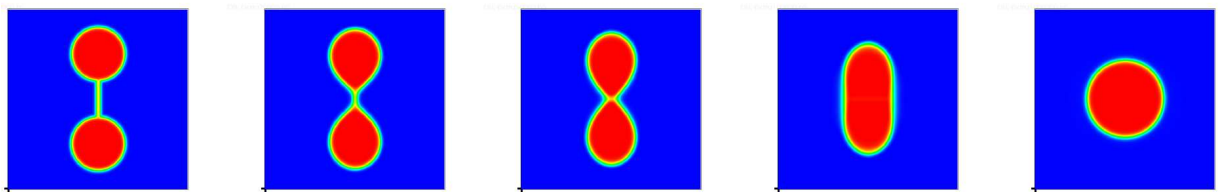

(d) PFV model, $\rho_{1}=10, \rho_{2}=1, t=0,2,5,10,150$
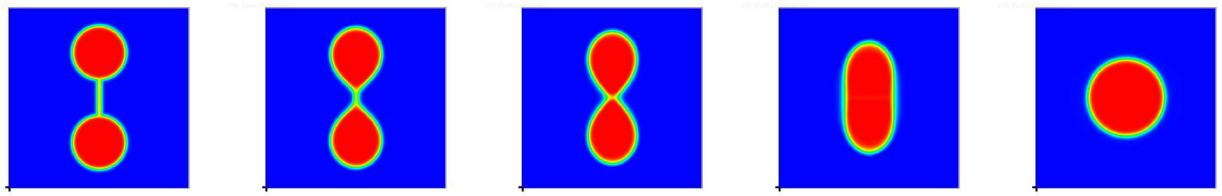

(e) PFV model, $\rho_{1}=1, \rho_{2}=10, t=0,2,5,10,150$

FIG. 3. Drop dynamics with respect to variable density ratios. (a-b) Results are obtained from the PFM model with $\rho_{1}=10, \rho_{2}=1 ; \rho_{1}=1, \rho_{2}=10$, respectively; (d-e) Results are obtained from the PFV model with $\rho_{1}=10, \rho_{2}=1 ; \rho_{1}=1, \rho_{2}=10$, respectively. When $\rho_{1}=\rho_{2}$, the PFV and the PFM models are identical. Here, we use the standard heat color map to plot the volume fraction of fluid 1, $\phi$, where red represents 1 and blue represents 0.

thus in the long term the energy drop is not as dramatic as that in the PFV model. In the equal density case and in the PFV model, the two drops eventually merge into a large drop so that the final energy level is lower. The drop dynamics is shown in Figure 3 and the energy decay curve as a function of time is shown in Figure 4.

When the density difference is not that large, the drop dynamics predicted by the PFM model resembles that predicted by the PFV model in that two drops are not clearly separated before they merge into a large one. See Figure 5 for the cases of 1:2, $2: 1,5: 1$, respectively. The case of $1: 5$ is similar to the case of $1: 10$ where two drops 


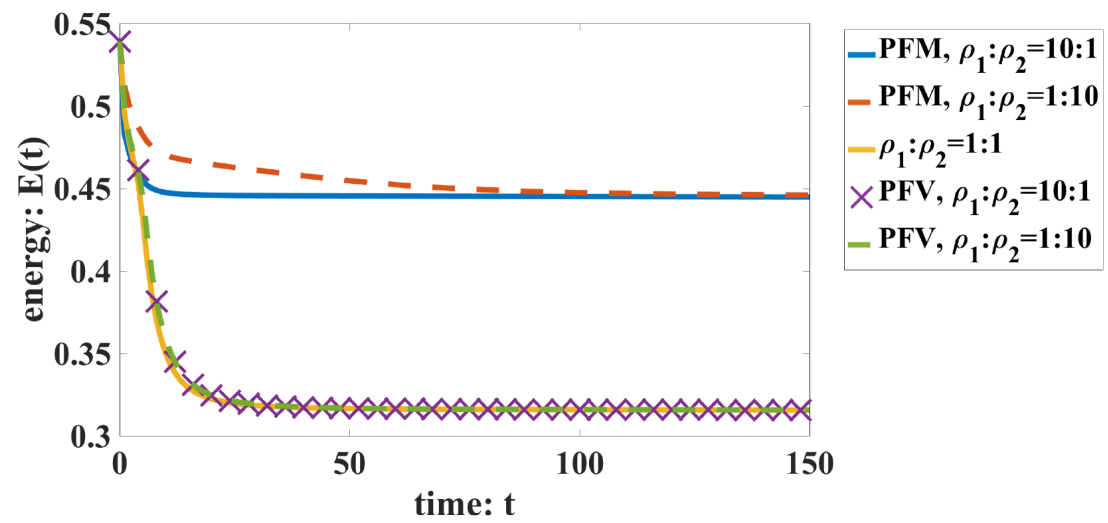

FIG. 4. Energy as a function of time for the drop dynamics simulation in Figure 3. The cases where the two drops coalesce into a large one show lower energy levels while the ones keeping separate drops show higher energy levels.
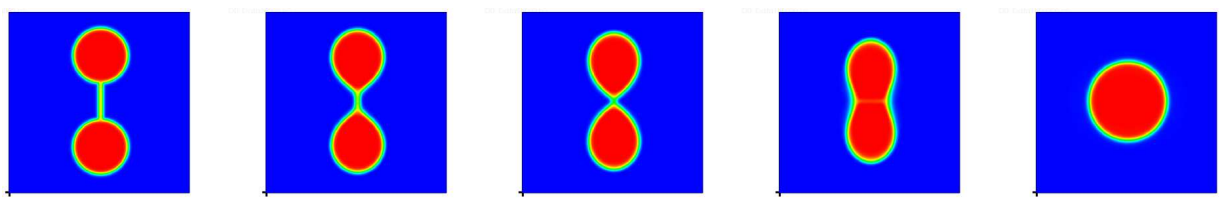

(a) PFM model, $\rho_{1}=1, \rho_{2}=2, t=0,2,5,10,150$
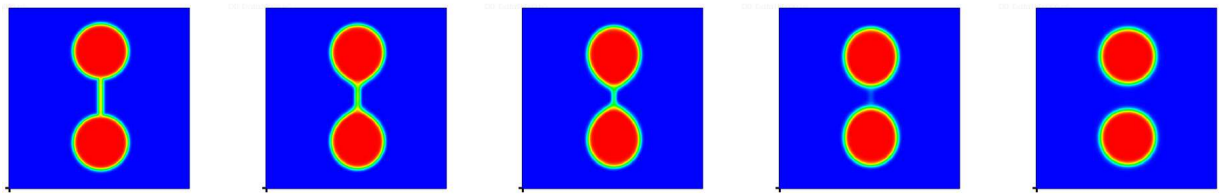

(b) PFM model, $\rho_{1}=1, \rho_{2}=5, t=0,2,5,10,150$
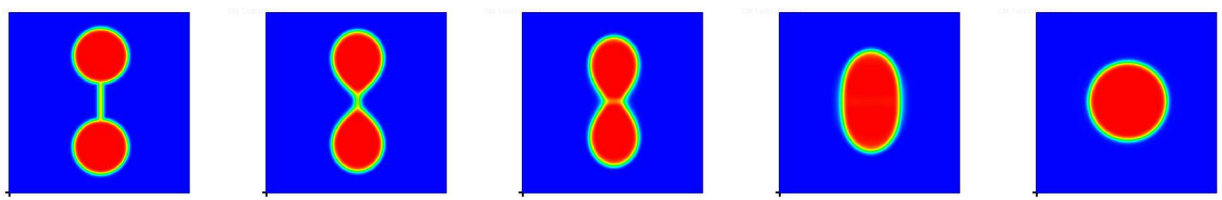

(c) PFM model, $\rho_{1}=2, \rho_{2}=1, t=0,2,5,10,150$
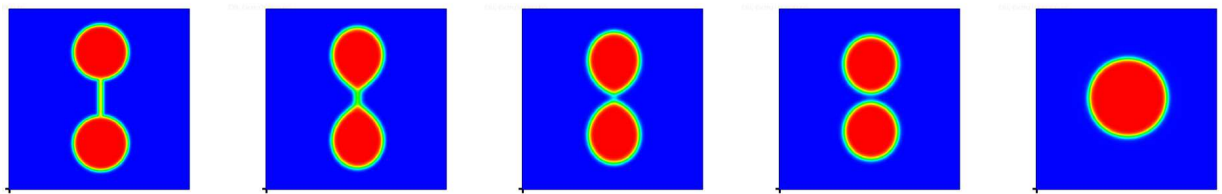

(d) PFM model, $\rho_{1}=5, \rho_{2}=1, t=0,2,5,10,150$

FIG. 5. Drop dynamics with the PFM model. This is the continuation from Figure 3. Here different density ratios are investigated for the PFM model alone. (a) $\rho_{1}: \rho_{2}=1: 2$; (b) $\rho_{1}: \rho_{2}=$ $1: 5$; (c) $\rho_{1}: \rho_{2}=2: 1$; (d) $\rho_{1}: \rho_{2}=5: 1$. At small density differences, small drops coalesce into a large one in the time frame we simulate. 


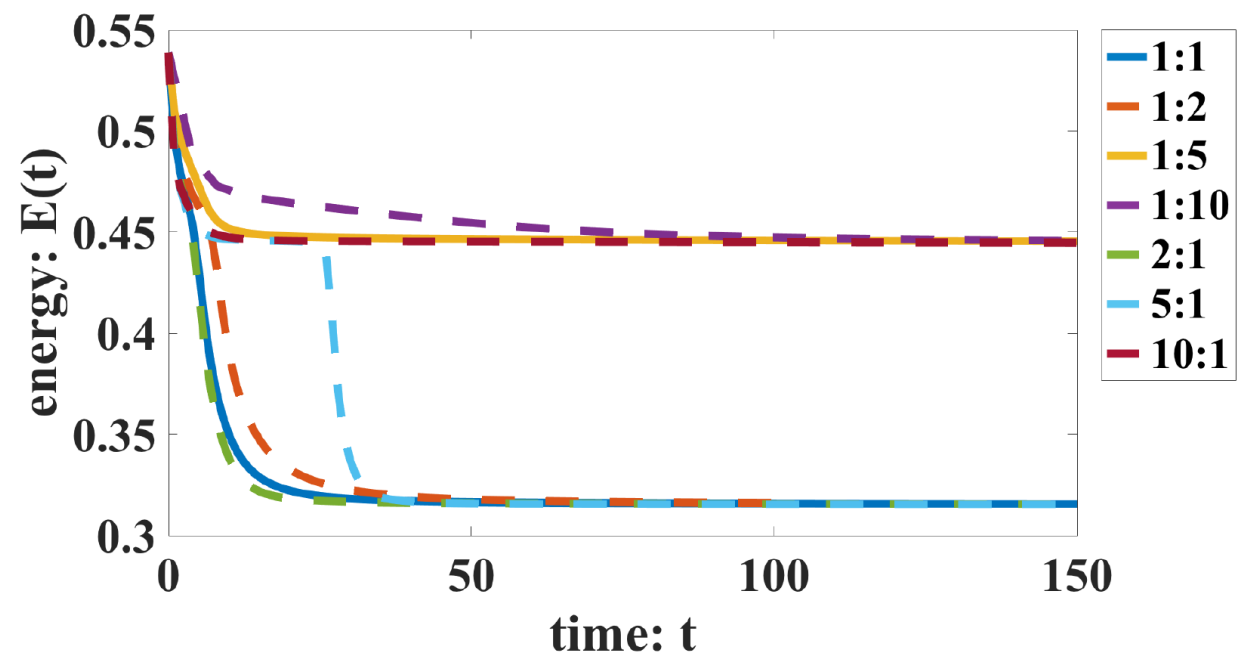

FIG. 6. Energy as a function of time for the drop dynamics simulation with the PFM model in Figures 3 and 5. The energy drops to the same level if one large drop forms at the end of the simulation. Analogously, the energy converges to the same value when two drops remain.

are clearly separated at the end of the computation $(t=150)$. Figure 6 depicts all the energy curves for the cases calculated using the PFM model up to $t=150$. Once again, the simulations reveal a strong dependence of the drop coalescent dynamics on the density difference in the PFM model.

Then, we use random initial conditions to study coarsening dynamics. Specifically, the initial profile is given by

$$
\phi=0.3+10^{-3} \operatorname{rand}(0,1) .
$$

The parameter values are chosen as

$$
\gamma_{1}=10^{-1}, \quad \gamma_{2}=10^{3}, \quad \eta_{1}=\eta_{2}=1, \quad \lambda=10^{-5}, \quad N=10^{7},
$$

and $L_{x}=L_{y}=1, N_{x}=N_{y}=128$. The velocity is initially assumed to be zero.

Here, we use the same initial profile of $\phi$ generated by the random number generator for all the runs, such that all the initial conditions in Figure 7 are identical, which is required to make the comparison meaningful. The numerical simulations are summarized in Figure 7 and the energy plot is shown in Figure 8. We observe that the PFM model with $\rho_{1}>\rho_{2}$ gives the fastest dynamics whereas the PFM model with $\rho_{1}<\rho_{2}$ gives the slowest dynamics. The PFV model yields similar dynamics for the two different density ratios, i.e., the dynamics predicted by PFV model is not sensitive to the density ratio. At the time we stop our computations, four results yield a similar energy level while the PVM model with $\rho_{1}>\rho_{2}$ shows a lower energy level than the other four.

5.3. Comparison of drop falling dynamics between the two models. In this subsection, we study transient dynamics of a heavy drop falling in the light fluid predicted by the two models comparatively. To consider the effect of gravity, an extra gravity force $\mathbf{F}$ is added in the momentum equation with

$$
\mathbf{F}=\rho g \hat{y}, \quad \rho=\rho_{1} \phi+\rho_{2}(1-\phi) .
$$



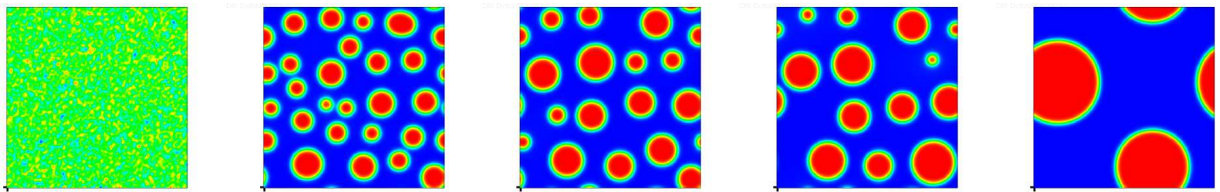

(a) PFM model, $\rho_{1}=10, \rho_{2}=1, t=0,1,5,10,35$
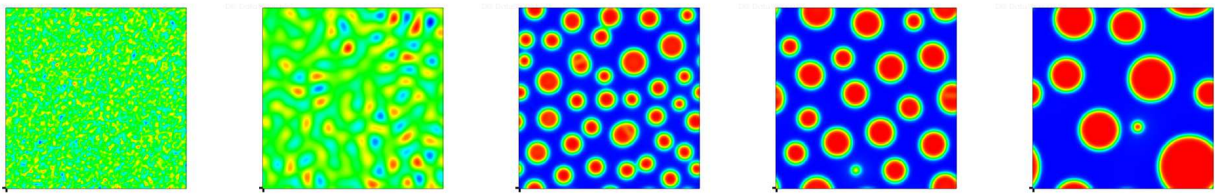

(b) PFM model, $\rho_{1}=1, \rho_{2}=10, t=0,1,5,10,100$
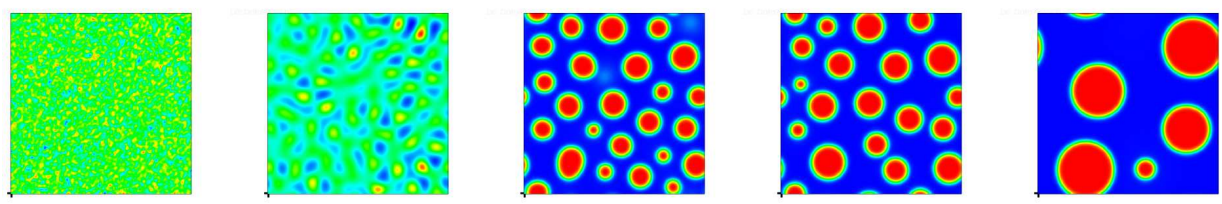

(c) $\rho_{1}=\rho_{2}=1, t=0,1,5,10,100$
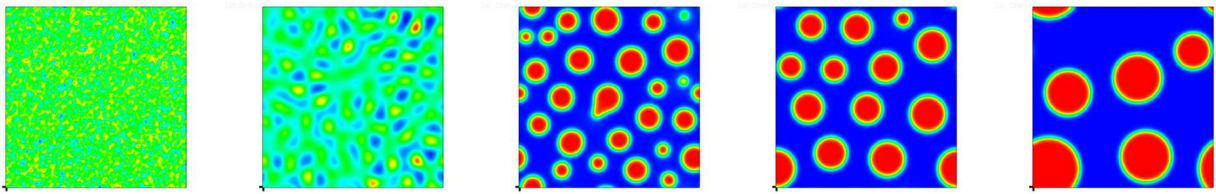

(d) PFV model, $\rho_{1}=1, \rho_{2}=10, t=0,1,5,10,100$
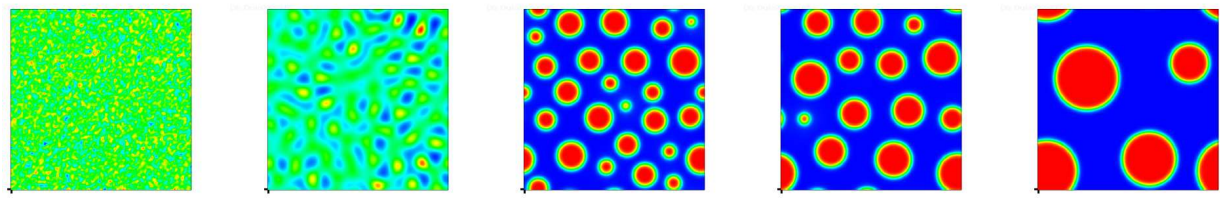

(e) PFV model, $\rho_{1}=10, \rho_{2}=1, t=0,1,5,10,100$

FIG. 7. Coarsening dynamics with a random initial condition. In this figure, we compare the coarsening dynamics between the two models at two sets of density ratios. The coarsening dynamics for the PFM model with density ratio $10: 1$ is the fastest.

The periodic boundary condition in the $x$ direction is used. In the $y$ direction, we implement the Dirichlet boundary condition for the velocity field $\left.\mathbf{v}\right|_{y=0}=\left.\mathbf{v}\right|_{y=L_{y}}=0$ and the Neumann boundary condition for $\phi$ :

$$
\left.\nabla \phi \cdot \mathbf{n}\right|_{y=0, y=1}=0,\left.\quad \nabla \mu \cdot \mathbf{n}\right|_{y=0, y=1}=0 .
$$

We remark that the modification of the boundary condition in the numerical solver invalidates our theoretical proof for energy stability of the numerical schemes with the periodic boundary condition. However, our numerical results indicate that the revised scheme is still energy stable. To solve the fully coupled linear system, we use the GMRES solver implemented in [8] with the preconditioner developed in [27] for the Navier-Stokes equation. 


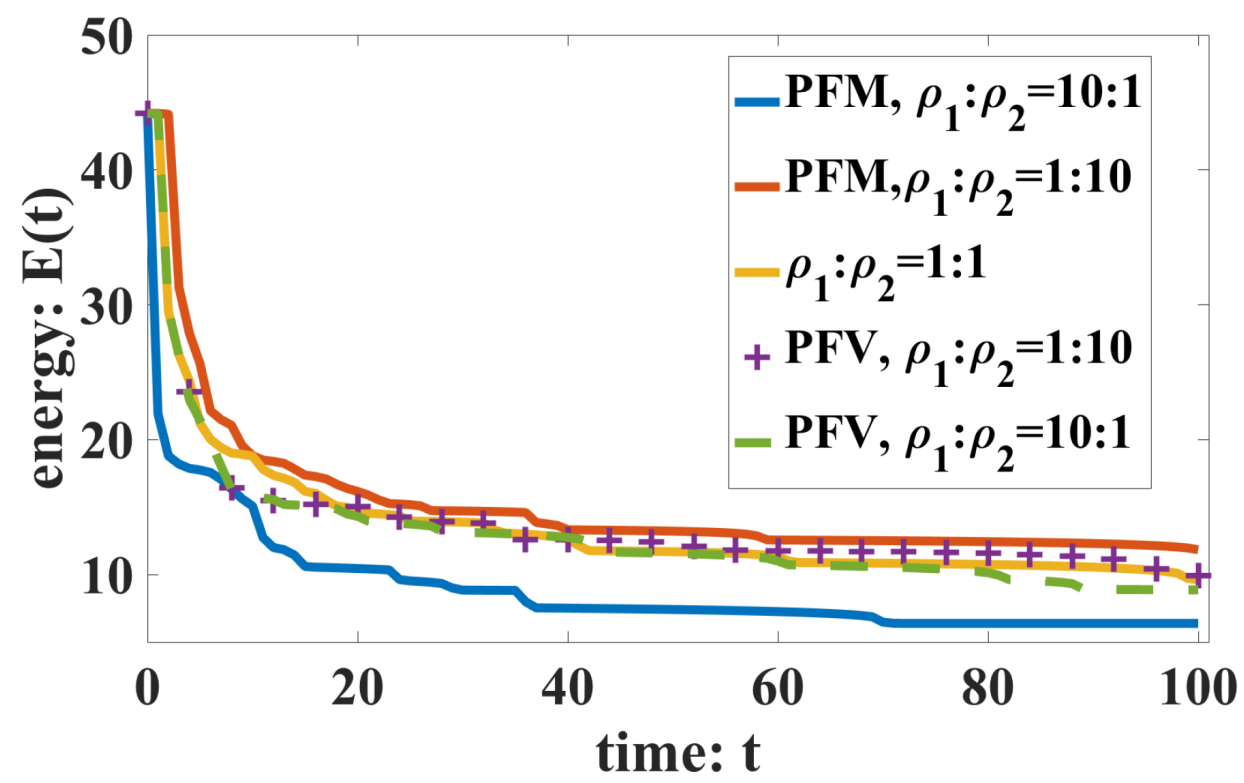

FIG. 8. Energy as a function of time for the coarsening dynamics in Figure 7.
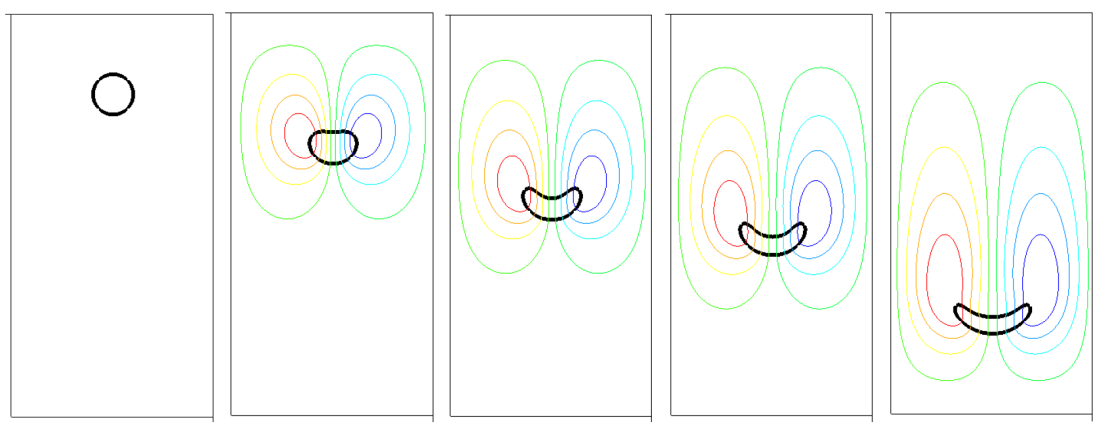

(a) PFM model, $\rho_{1}=5, \rho_{2}=1, t=0,0.5,0.8,1,1.5$
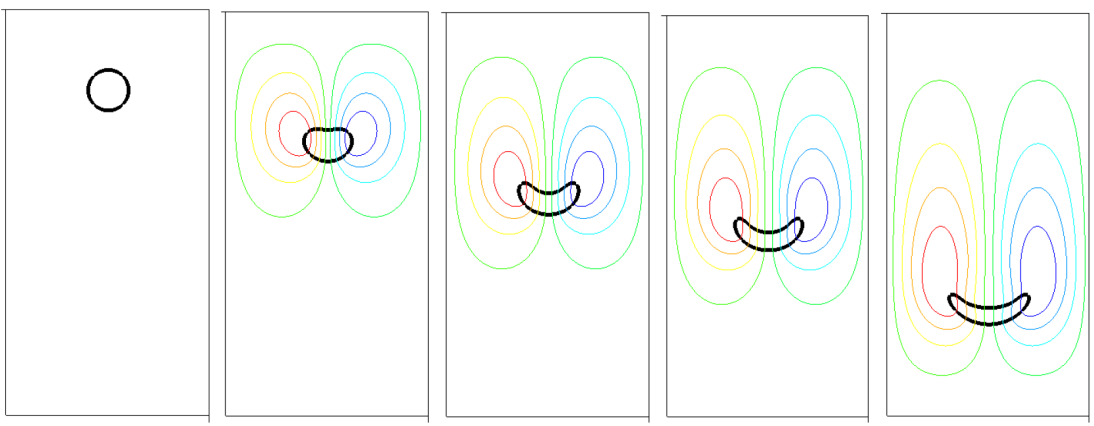

(b) PFV model, $\rho_{1}=5, \rho_{2}=1, t=0,0.5,0.8,1,1.5$

Fig. 9. Heavy fluid drop falling in a light fluid matrix. Here, we use $\rho_{1}=5, \rho_{2}=1$. The phase boundary defined by $\phi=0.5$ is plotted with a thick black line. The color circles are the streamlines. (a) Numerical results at $t=0,0.5,0.8,1,1.5$ for the PFM model are shown, respectively. (b) Numerical results at $t=0,0.5,0.8,1,1.5$ for the PFV model are depicted, respectively. They predict comparable drop shapes when density difference is small.

Copyright (c) by SIAM. Unauthorized reproduction of this article is prohibited. 

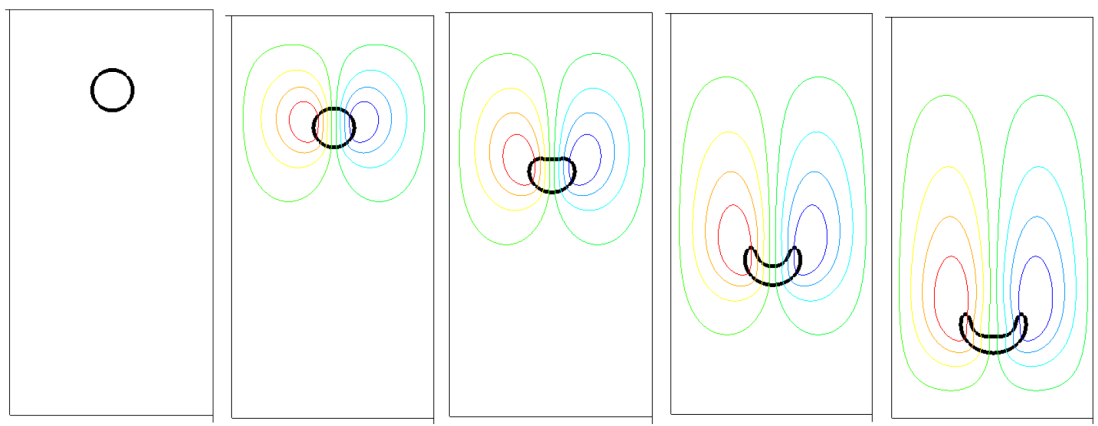

(a) PFM model, $\rho_{1}=10, \rho_{2}=1, t=0,0.3,0.5,0.8,1$
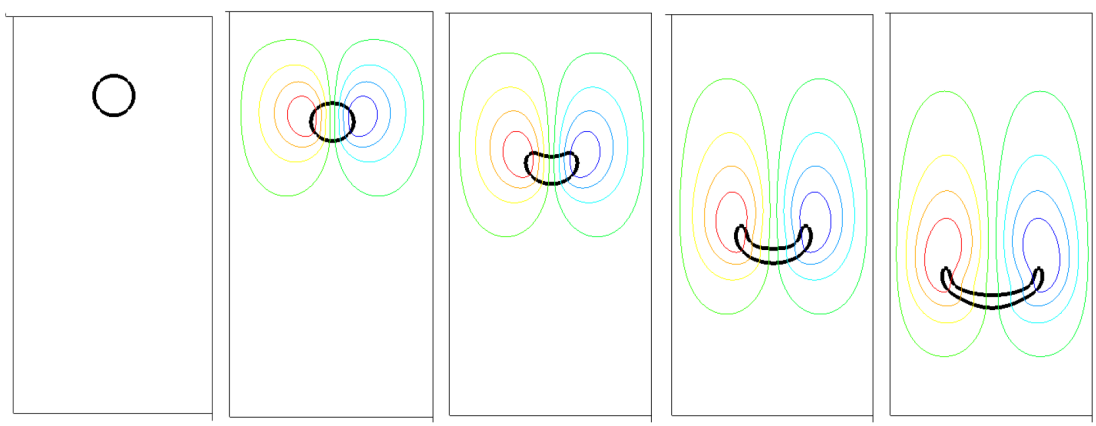

(b) PFV model, $\rho_{1}=10, \rho_{2}=1, t=0,0.3,0.5,0.8,1$

FIG. 10. Heavy fluid drop falling in a light fluid matrix. Here, we use $\rho_{1}=10, \rho_{2}=1$. The phase boundary defined by $\phi=0.5$ (i.e., the interface) is plotted with a thick black line. The streamlines are shown as colored circles. (a) The PFM model prediction at $t=0,0.3,0.5,0.8,1$, respectively. (b) The PFV model prediction at $t=0,0.3,0.5,0.8,1$, respectively. The two models predict different shapes for the drop at later time.

We use $L_{x}=2, L_{y}=4, N_{x}=256, N_{y}=512$, and the parameter values are chosen as

$$
\eta_{1}=\eta_{2}=0.1, \quad \gamma_{1}=10^{-2}, \quad \gamma_{2}=10^{2}, \quad \lambda=10^{-4}, \quad g=9.8
$$

The initial profile is chosen as

$$
\phi=\frac{1}{2}\left(1+\tanh \frac{0.2-R}{0.01}\right), \quad R=\sqrt{\left(y-0.8 L_{y}\right)^{2}+\left(x-0.5 L_{x}\right)^{2}}, \quad \mathbf{v}=0 .
$$

Here, we conduct two simulations with $\rho_{1}=5, \rho_{2}=1$ shown in Figure 9 and $\rho_{1}=$ 10, $\rho_{2}=1$ shown in Figure 10, respectively. The vorticity, velocity, pressure field, and divergence of velocity for Figure 10 are shown in Figure 11. We observe that when the density difference is small, the two models predict qualitatively similar dynamics. However, when the density difference is large, shapes of the falling drop are quite different. The corresponding velocity fields are also shown to be different.

6. Concluding remarks. In this paper, we present a novel linearization (energy quadratization) strategy to develop two spatial-temporal, second order, fully discrete, linear schemes for a quasi-incompressible model and an incompressible model of a binary viscous fluid mixture of different densities. Mesh refinements are conducted to verify the accuracy of the new schemes. The fully discrete linear schemes are shown to be unconditionally energy stable with respect to periodic boundary conditions such that a relatively large time step is plausible. As a corollary, the time-discretized, 


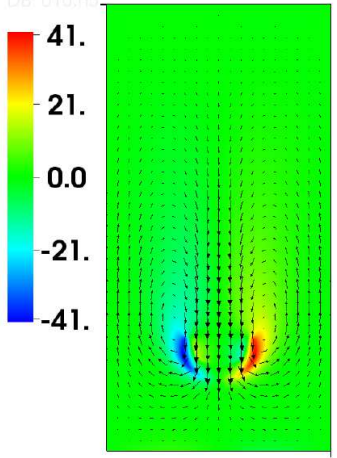

(a) Vorticity

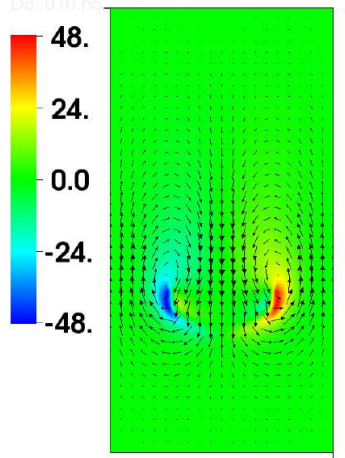

(d) Vorticity

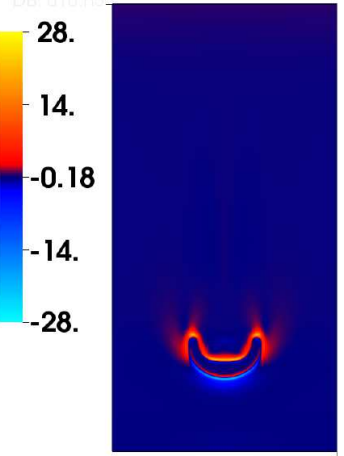

(b) $\nabla \cdot \mathbf{v}$

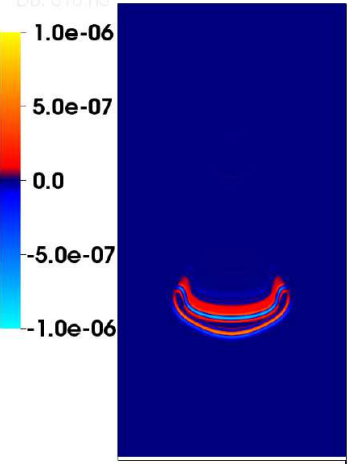

(e) $\nabla \cdot v$

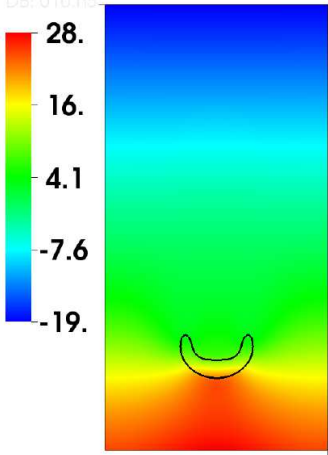

(c) Pressure

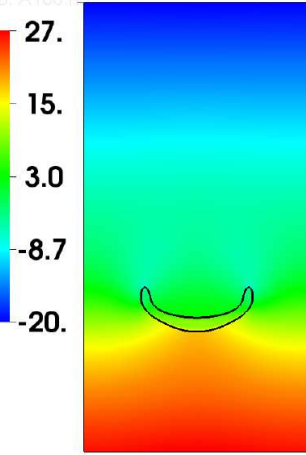

(f) Pressue

FIG. 11. Vorticity, divergence of velocity field $(\nabla \cdot \mathbf{v})$, and pressure for simulations in Figure 10 at $t=1$. The arrows in (a) and (c) represents the velocity field. (a-c) vorticity, divergence of velocity field, and pressure at $t=1$ for Figure 10(a) with the PFM model; (d)-(f) vorticity, divergence of velocity field, and pressure at $t=1$ for Figure 10(b) with the PFV model.

semidiscrete schemes are also unconditionally energy stable, subject to appropriate physical boundary conditions. Several numerical results are presented to illustrate the efficiency of our numerical schemes. The linearization idea is rather general and useful so that it can be applied to study a broad class of hydrodynamic models as well. The upshot of the numerical studies is that the two models predict qualitatively similar dynamics when density difference is small; however, they yield quite different dynamics when the density difference is large. In the latter case, we believe the quasiincompressible model is more accurate physically since the linear momentum is not conserved in the incompressible model.

\section{REFERENCES}

[1] H. ABELS, Existence of weak solutions for a diffuse interface model for viscous, incompressible fluids with general densities, Commun. Math. Phys., 289 (2009), pp. 45-73.

[2] H. ABELS, Strong well-posedness of a diffuse interface model for a viscous, quasi-incompressible two-phase flow, SIAM J. Math. Anal., 44 (2012), pp. 316-340.

[3] H. Abels And H. Garcke, Thermodynamically consistent frame indifferent diffuse interface models for impressible two phase flows with different densities, Math. Models Methods Appl. Sci., 22 (2012), p. 1150013. 
[4] S. W. A. E. Diegel And X. H. Feng, Analysis of a mixed finite element method for a CahnHilliard-Darcy-Stokes system, SIAM J. Numer. Anal., 53 (2015), pp. 127-152.

[5] G. L. Aki, W. Dreyer, J. Giesselmann, and C. Kraus, A quasi-incompressible diffuse interface model with phase transition, Math. Models Methods Appl. Sci., 24 (2014), pp. 827-861.

[6] S. Aland AND A. Voigt, Benchmark computations of diffuse interface models for twodimensional bubble dynamics, Int. J. Numer. Methods Fluids, 69 (2012), pp. 747-761.

[7] V. E. Badalassi, H. D. Ceniceros, and S. BanerJee, Computation of multiphase systems with phase field models, J. Comput. Phys., 190 (2003), pp. 371-397.

[8] N. Bell And M. Garland, Cusp: Generic parallel algorithms for sparse matrix and graph computations, Version 0.3. 0, 35, 2012.

[9] R. Borcia AND M. Bestehorn, Phase-field model for Marangoni convection in liquid-gas systems with a deformable interface, Phys. Rev. E, 67 (2003), p. 066307.

[10] F. BOYER, A theoretical and numerical model for the study of incompressible mixture flows, Comput. Fluids, 31 (2002), pp. 41-68.

[11] R. Chella and J. Vinals, Mixing of a two-phase fluid by cavity flow, Phys. Rev. E, 53 (1996), pp. $3832-3840$.

[12] L. Q. Chen, Phase-field models for microstructure evolution, Ann. Rev. Mater. Res., 32 (2002), pp. 113-140.

[13] W. Chen, W. Feng, Y. Liu, C. Wang, and S. Wise, A Second Order Energy Stable Scheme for the Cahn-Hilliard-Hele-Shaw Equations, preprint, http://arxiv.org/abs/1611.02967, 2016.

[14] W. Chen, Y. Liu, C. Wang, And S. Wise, Convergence analysis of a fully discrete finite difference scheme for the Cahn-Hilliard-Hele-Shaw equation, Math. Comput., 85 (2016), pp. 2231-2257.

[15] C. Collins, J. Shen, And S. Wise, An efficient, energy stable scheme for the Cahn-HilliardBrinkman system, Commun. Comput. Phys., 13 (2013), pp. 929-957.

[16] A. Diegel, C. Wang, X. Wang, and S. Wise, Convergence Analysis and Error Estimates for a Second Order Accurate Finite Element Method for the Cahn-Hilliard-Navier-Stokes System, preprint, http://arxiv.org/abs/1606.02668, 2016.

[17] H. Ding, P. D. M. Spelt, And C. Shu, Diffuse interface model for incompressible two-phase flows with large density ratios, J. Comput. Phys., 226 (2007), pp. 2078-2095.

[18] M. DoI, Introduction to Polymer Physics, Oxford University Press, Oxford, 1996.

[19] Q. Du, C. Liu, AND X. WANG, A phase field approach in the numerical study of the elastic bending energy for vesicle membranes, J. Comput. Phys., 198 (2004), pp. 450-468.

[20] Q. Du, C. LIU, AND X. WANG, Retrieving topological information for phase field models, SIAM J. Appl. Math., 65 (2005), pp. 1913-1932.

[21] P. J. FLORY, Principles of Polymer Chemistry, Cornell University Press, Ithaca, NY, 1953.

[22] H. Garcke, M. Hinze, and C. Kahle, A stable and linear time discretization for a thermodynamically consistent model for two-phase incompressible flow, Appl. Numer. Math., 99 (2016), pp. 151-171.

[23] J. Giesselmann And T. Pryer, Energy consistent discontinuous Galerkin methods for a quasiincompressible diffuse two phase flow model, ESAIM Math. Model. Numer. Anal., 49 (2015), pp. 275-301.

[24] Y. Gong, X. LiU, AND Q. WAng, Fully discretized energy stable schemes for hydrodynamic equations governing two-phase viscous fluid flows, J. Sci. Comput., 69 (2016), pp. 921-945.

[25] Y. Gong, J. ZhaO, AND Q. WANG, An energy stable algorithm for a quasi-incompressible hydrodynamic phase-field model of viscous fluid mixtures with variable densities and viscosities, Comput. Phys. Commun., 219 (2017), pp. 20-34.

[26] Y. Gong, J. ZhaO, AND Q. WANG, Linear second order in time energy stable schemes for hydrodynamic models of binary mixtures based on a spatially pseudospectral approximation, Adv. Comput. Math., to appear.

[27] B. E. GRIFFith, An accurate and efficient method for the incompressible Navier-Stokes equations using the projection method as a preconditioner, J. Comput. Phys., 228 (2009), pp. $7565-7595$.

[28] G. GRÜN, On convergent schemes for diffuse interface models for two-phase flow of incompressible fluids with general mass densities, SIAM J. Numer. Anal., 51 (2013), pp. 3036-3061.

[29] G. GRün And F. KlingbeIL, Two-phase flow with mass density contrast: Stable schemes for a thermodynamic consistent and frame-indifferent diffuse-interface model, J. Comput. Phys., 257 (2014), pp. 708-725.

[30] Z. Guo, P. Lin, and J. Lowengrub, A numerical method for the quasi-incompressible CahnHilliard-Navier-Stokes equations for variable density flows with a discrete energy law, J. Comput. Phys., 276 (2014), pp. 486-50s.

Copyright (c) by SIAM. Unauthorized reproduction of this article is prohibited. 
[31] Z. Guo, P. Lin, J. Lowengrub, And S. Wise, Mass conservative and energy stable finite difference methods for the quasi-incompressible Navier-Stokes-Cahn-Hilliard system: Primitive variable and projection-type schemes, Comput. Methods Appl. Mech. Engrg., 326 (2017), pp. $144-174$.

[32] Z. GuO, P. Lin, AND Y. WANG, Continuous finite element schemes for a phase field model in two-layer fluid Benard-Marangoni convection computations, Comput. Phys. Commun., 185 (2014), pp. 63-78.

[33] M. E. Gurtin, D. Polignone, And J. Vinals, Two-phase binary fluids and immiscible fluids described by an order parameter, Math. Models Methods Appl. Sci., 6 (1996), pp. 815-831.

[34] P. C. Hohenberg And B. I. Halperin, Theory of dynamic critical phenomena, Rev. Mod. Phys., 49 (1977), pp. 435-479.

[35] T. Y. Hou, J. S. Lowengrub, And M. J. Shelley, Boundary integral methods for multicomponent fluids and multiphase materials, J. Comput. Phys., 169 (2001), pp. 302-362.

[36] J. Hua, P. Lin, C. Liu, ANd Q. WANG, Energy law preserving $C^{0}$ finite element schemes for phase field models in two-phase flow computations, J. Comput. Phys., 230 (2011), pp. $7115-7131$.

[37] D. JaCQmin, Calculation of two-phase Navier-Stokes flows using phase-field modeling, J. Comput. Phys., 155 (1999), pp. 96-127.

[38] J. Kim And J. Lowengrub, Phase field modeling and simulation of three-phase flows, Interfaces Free Bound., 7 (2005), pp. 435-466.

[39] J. Li AND Q. WANG, A class of conservative phase field models for multiphase fluid flows, J. Appl. Mech., 81 (2014), p. 021004.

[40] C. Liu And J. Shen, A phase field model for the mixture of two incompressible fluids and its approximation by a Fourier-spectral method, Physica D, 179 (2003), pp. 211-228.

[41] J. Lowengrub, A. Ratz, And A. Voigt, Phase field modeling of the dynamics of multicomponent vesicles spinodal decomposition coarsening budding and fission, Phys. Rev. E, 79 (2009).

[42] J. S. Lowengrub and L. Truskinovsky, Quasi incompressible Cahn-Hilliard fluids and topological transitions, Proc. Roy. Soc. A, 454 (1998), pp. 2617-2654.

[43] R. Mittal and G. Iaccarino, Immersed boundary methods, Ann. Rev. Fluid Mech., 37 (2005), pp. 239-261.

[44] J. Shen AND X. YANG, A phase-field model for two-phase flows with large density ratio and its numerical approximation, SIAM J. Sci. Comput., 32 (2010), pp. 1159-1179.

[45] J. Shen, X. YAnG, AND Q. WAng, Mass and volume conservation in phase field models for binary fluids, Commun. Comput. Phys., 13 (2013), pp. 1045-1065.

[46] K. E. Teigen, P. Song, J. Lowengrub, and A. Voigt, A diffuse-interface method for twophase flows with soluble surfactants, J. Comput. Phys., 230 (2011), pp. 375-393.

[47] G. Tryggrason, B. Bunner, A. Esmaeeli, D. Juric, N. Al-Rawahi, W. Tauber, J. Han, S. NAS, AND Y. J. JAN, A front-tracking method for the computations of multiphase flow, J. Comput. Phys., 169 (2001), pp. 708-759.

[48] M. Verschueren, F. N. V. De Vosse, and H. E. H. Meijer, Diffuse-interface modelling of thermocapillary flow instabilities in a Hele-Shaw cell, J. Fluid Mech., 434 (2001), pp. 153-166.

[49] S. Wise, J. Lowengrub, H. Frieboes, and B. Cristini, Three dimensional multispecies nonlinear tumor growth I: Model and numerical method, J. Theor. Biol., 253 (2008), pp. 524-543.

[50] X. YANG, Linear, first- and second-order, unconditionally energy stable numerical schemes for the phase field model of homopolymer blends, J. Comput. Phys., 327 (2016), pp. 294-316.

[51] X. YANG, Numerical approximations for the Cahn-Hilliard phase field model of the binary fluidsurfactant system, J. Sci. Comput., https://doi.org/10.1007/s10915-017-0508-6 (2017).

[52] X. Yang, M. G. Forest, AND Q. WANG, Near equilibrium dynamics and one-dimensional spatial-temporal structures of polar active liquid crystals, Chin. Phys. B, 23 (2014), p. 118701.

[53] X. YANG AND L. JU, Efficient linear schemes with unconditional energy stability for the phase field elastic bending energy model, J. Comput. Phys., 315 (2017), pp. 691-712.

[54] X. YANG AND L. JU, Linear and unconditionally energy stable schemes for the binary fluid-surfactant phase field model, Comput. Methods. Appl. Mech. Engrg., 318 (2017), pp. 1005-1029.

[55] X. YAnG, Y. GONG, J. Li, J. ZhaO, AND Q. WANG, On hydrodynamic phase field models for binary fluid mixture, Theor. Comp. Fluid Dyn., (2017).

[56] X. YANG, J. ZhaO, AND Q. WANG, Numerical approximations for the molecular beam epitaxial growth model based on the invariant energy quadratization method, J. Comput. Phys., 333 (2017), pp. 102-127.

Copyright (c) by SIAM. Unauthorized reproduction of this article is prohibited. 
[57] X. YAng, J. Zhao, Q. WAng, And J. Shen, Numerical approximations for a three components cahn-hilliard phase-field model based on the invariant energy quadratization method, Math. Models Methods Appl. Sci., 27 (2017), pp. 1993-2023.

[58] P. Yue, J. Feng, C. Liu, And J. Shen, A diffuse-interface method for simulating two-phase flows of complex fluids, J. Fluid. Mech., 515 (2004), pp. 293-317.

[59] J. Zhao, P. Seeluangsawat, and Q. WAng, Modeling antimicrobial tolerance and treatment of heterogeneous biofilms, Math. Biosci., 282 (2016), pp. 1-15.

[60] J. Zhao, Y. Shen, M. Happasalo, Z. J. Wang, and Q. Wang, A 3 D numerical study of antimicrobial persistence in heterogeneous multi-species biofilms, J. Theor. Biol., 392 (2016), pp. 83-98.

[61] J. ZhaO AND Q. WANG, Modeling cytokinesis of eukaryotic cells driven by the actomyosin contractile ring, Int. J. Numer. Methods Biomed. Eng., 32 (2016), p. e02774.

[62] J. ZHAO AND Q. WANG, Three-dimensional numerical simulations of biofilm dynamics with quorum sensing in a flow cell, Bull. Math. Biol., 79 (2017), pp. 884-919.

[63] J. ZhaO, Q. WANG, AND X. YANG, Numerical approximations to a new phase field model for two phase flows of complex fluids, Comput. Methods Appl. Mech. Engrg., 310 (2016), pp. $77-97$.

[64] J. ZHAO, Q. WANG, AND X. YANG, Numerical approximations for a phase field dendritic crystal growth model based on invariant energy quadratization, Int. J. Numer. Methods Engrg., 110 (2017), pp. 279-300.

[65] J. Zhao, X. YAng, Y. Gong, And Q. WAng, A novel linear second order unconditionally energy-stable scheme for a hydrodynamic $q$ tensor model for liquid crystals, Comput. Methods Appl. Mech. Engrg., 318 (2017), pp. 803-825.

Copyright (c) by SIAM. Unauthorized reproduction of this article is prohibited. 